\title{
Incorporating a Bayesian Network into Two-Stage Stochastic Programming for Blood Bank Location-Inventory Problem in Case of Disasters
}

\author{
Shutong Chen (iD) and Changjun Wang (D) \\ Glorious Sun School of Business and Management, Donghua University, No. 1882 West Yarian Road, Shanghai 200051, China
}

Correspondence should be addressed to Changjun Wang; cjwang@dhu.edu.cn

Received 7 September 2018; Revised 27 January 2019; Accepted 11 February 2019; Published 7 March 2019

Academic Editor: Lu Zhen

Copyright (C) 2019 Shutong Chen and Changjun Wang. This is an open access article distributed under the Creative Commons Attribution License, which permits unrestricted use, distribution, and reproduction in any medium, provided the original work is properly cited.

\begin{abstract}
This paper is concerned with the optimal decisions of blood banks in a blood logistics network (BLN) with the consideration of natural disasters. One of the biggest challenges is how to deal with unexpected disasters. Our idea is to consider the disasters as the natural consequences of interaction among multiple interdependent uncertain factors, such as the locations and the levels of disasters, the number of casualties, and the availabilities of rescue facilities, which work together to influence the rescue effects of the BLN. Thus, taking earthquakes as the example, a Bayesian Network is proposed to describe such uncertainties and interdependences and, then, we incorporate it into a dedicated two-stage multi-period stochastic programming model for the BLN. The planning stage in the model focuses on blood bank location and inventory decisions. The subsequent operational stage is composed of multiple periods, some of which may suffer disasters and initiate corresponding rescue operations. Numerical tests show that the proposed approach can be efficiently applied in blood management under the complicated disaster scenarios.
\end{abstract}

\section{Introduction}

The supply of human blood relies on Blood Logistics Networks (BLNs), which are usually composed of donor points, blood banks, and relief facilities, etc. As the facilities to collect, test, inspect, store, and distribute human blood, blood banks play a fundamental role in BLNs to ensure the effective and efficient supply. Cooperating with other facilities in BLNs, blood banks should satisfy not only the daily demand of human blood but also the emergency demand caused by natural and anthropogenic disasters. Especially in recent years, frequent occurrences of disasters, such as earthquakes, hurricanes, fires, and terrorist attacks, have caused significant personnel and property losses due to its uncertain and destructive nature [3]. A well-designed blood bank in the BLN, which includes the prepositioning of emergency aid before a disaster strike and the supply of the human blood during a response process, can effectively overcome the suddenness of disasters.
However, the blood banks related decisions are always fraught with different challenges, in which the first and most important one is the availability of the disaster information that is indispensable to make the decisions. Taking the demand on human blood as example, the daily demand may be stable. However, the emergency demand in a disaster, especially the suddenly-occurring disaster such as the earthquake in natural disasters or terrorist attack, is hard to be estimated using historical data directly [4]. Besides the demand, other stochastic events in disasters, such as the place where the disaster occurs and facility failure, which also influence the relief effects significantly, would bring extra difficulties to decision-making. Moreover, the decisions on the blood banks include not only the strategic issues by considering the locations, but also the operational activities, such as inventory control and distribution. The interaction between the strategic level and the operational level requires an integrated model at the whole BLN level, taking the costs of facility location, transportation, and inventory into account 
[5]. Finally, human blood has its distinctive characteristics, such as multiple categories (e.g., red blood cells, platelets, white blood cells, etc.) with different blood types (type A, $\mathrm{B}, \mathrm{AB}$, and $\mathrm{O}$ ) and the mutual substitution between various types [6]. For example, type $O$ could serve as the substitution of blood types A, B, and AB [7].

Some researchers have adopted the integrated framework to study relief logistics network taking disasters into account. However, few works have addressed blood banks with planning stage and multiple operational stages simultaneously as well as the features of blood products. More importantly, the uncertainties of disasters are dealt with in a relatively simple way in previous BLN-related studies. Specifically, besides daily demand, blood banks should also offer emergency aid to the hospitals in case of disasters. Demand uncertainty is a crucial challenge that must be handled in disaster management [8]. To handle this challenge, the uncertain demand for relief goods caused by the disasters is usually assumed available in many studies [9]. Such assumption may be too strict, especially for suddenly-occurring disasters like earthquakes. Actually, besides the uncertain demand, each disaster usually contains multiple sources of uncertainty, such as the magnitude of the disaster, the number of casualties, and the availability of rescue facilities, which should be considered when we design an efficient emergency plan [10]. It is worth noting that these factors are mutually interdependent. For example, the higher magnitude of earthquake in some places is, the more casualties there are, the higher failure probability a local hospital has, and finally the more human blood is required. Thus, it inspires us to consider more stochastic factors involved in disasters and focus on how these factors interact with each other and contribute together to the final disaster consequences. With the consideration of these random factors, the decisions we study would be more comprehensive and reasonable. However, it also means a dedicated mathematical programming model is required. For example, if the failure of rescue facilities, which often occurs in disasters, is considered, we should design the corresponding model which can handle this case and make appropriate decisions on blood allocation.

Therefore, our study mainly deals with the locationinventory decisions on blood banks and related decisions in the BLN with the consideration of possible occurrences of disasters. We propose a two-stage multi-period Stochastic Programming (SP) model which takes the characteristics of human blood (such as multiple blood products, blood lifespan, and blood substitution) and multiple stochastic factors involved in disasters into account. In the planning stage of the model, we determine the blood bank location and the inventory levels in the selected blood bank and each hospital in the BLN. The following operational stage involves multiple periods, in which the distribution and replenishment decisions on human blood would be made periodically. When a disaster occurs in some periods, the BLN should have the ability to continuously satisfy the emergency blood transfusion requirements at the first-time treatment by its inventory. The corresponding transfusion quantity, as one of the consequences of disasters, is decided by multiple disasterrelated factors, such as the magnitude of the disaster, the number of the injured, the level of injury, and the distribution of blood type among the injured. Besides, some regionrelated stochastic factors, such as the potential disaster points and the unavailable probability of each hospital, would decide which hospital provides continuous blood supply during a transfusion time, and, then, influence the prepositioning inventory-level decisions in this rescue hospital because there is a replenishment time due to its distance from the blood bank. Thus, we apply the Bayesian Network (BN), which is a probabilistic modelling approach in the context of data mining, to synthesize all these random factors to generate discrete scenarios which can be used to describe the possible disaster. The integrated objective is given to optimize the total cost of the strategic and operational decisions of the whole BLN during a planning horizon. A case study based on earthquakes in Sichuan Province, China, is studied and the corresponding model, which is a mixedinteger linear program, is optimally solved by IBM ILOG CPLEX to demonstrate the effectiveness of the proposed methods.

The contribution of this study lies in two aspects. Firstly, we propose a $\mathrm{BN}$ model to utilize the interdependences of multiple uncertain factors to generate plausible scenarios and incorporate it into a dedicated optimization model. It allows us to synthesize multiple stochastic elements in disasters and study their aggregated impacts on rescue management. To the best of our knowledge, a modelling effort for location and inventory in BLNs considering BN-based scenarios generation does not appear in existing studies. Our work is a new attempt to combine optimization method with the technique from data science to address a complicated decision-making issue in real-world. Secondly, we formulate an integrated SP model to handle with the location decision on blood banks and related inventory as well as distribution decisions in the BLN with possible disasters. The aforementioned features (e.g., coordination of location and operational activities in the whole BLN, the typical characteristics of human blood, and multiple uncertain factors considered in the $\mathrm{BN}$ ) are considered in our model. In previous works on the BLNs, the consideration of some features of human blood (e.g., multiple blood products, blood substitution) is limited. Also, few BLNrelated papers consider multiple interdependent uncertain factors, some of which are not studied as we know, such as the blood type among the injured and the level of injury. Thus, we take an early step to incorporate both multiple random factors and unique characteristics of blood into blood management considering disasters.

The remainder of this study is presented as follows. Section 2 reviews some of the more recent and related articles on correlative areas. In Section 3, a two-stage multiperiod SP model is developed, and the corresponding $\mathrm{BN}$ is introduced to quantify the effect of multiple sources of uncertainty on scenarios. An application in earthquake crisis faced by Sichuan Province, China, is given in Section 4 and the sensitivity analysis is implemented to validate the proposed model. The Expected Value (EV) model is given in Section 5 to prove the value of the stochastic solution. The last section presents conclusions and remarks on some directions for future research. 


\section{Literature Review}

In this section, the literature regarding relief logistics networks under disasters and the applications of Bayesian Networks (BNs) are unfolded as follows.

Recently, frequent-occurring disasters drive researchers to study relief logistics networks with the aim of mitigating the impacts of disasters. In a disaster, since two stages exist naturally, i.e., pre/postdisaster, most of the existing studies formulate two-stage models to study the related issues of relief logistics networks. Barbarosoğlu and Arda's work [11] is the pioneer study which models predisaster and postdisaster stages by a two-stage SP and plans the transportation of relief goods in case of disaster. Sequentially, some researchers focus on the joint location-inventory problems (e.g., [12$14])$, location-distribution problems (e.g., $[8,15])$, locationrouting problems $[12,16]$, etc. under disasters based on the two-stage paradigm, in which multiple sources of disaster uncertainties like demand, transportation time, and facility disruption are considered. However, most existing studies handle these uncertainties independently. For example, Mete and Zabinsky [12] determine the location of storage points from possible warehouses and the required inventory levels for medical supplies in the first stage with the consideration of uncertain transportation time and demand in the second stage. A multiobjective SP model is proposed by Haghi et al. [13] for determining the locations of distribution centers (DCs) and health centers, as well as the inventory of relief goods stored by suppliers before a disaster. Random demand, the number of casualties, and uncertain transportation time are considered independently in scenarios. Mohamadi and Yaghoubi [15] address the location of relief facilities in which several random factors, such as the number of injured people, the failure probabilities of roads, and the travel time, are also separately considered.

Besides, only a few authors have incorporated the interdependences between random disaster-related factors within humanitarian operations. For example, Verma and Gaukler [17] study the location decision by two-stage SP. Their contribution is a distance-damage function, which is used to model the damage caused by the disaster, and thus corresponding scenarios could be constructed. To determine the locations and capacities of DCs, Paul and MacDonald [10] treat the magnitude as an original stochastic factor, making the facility damage and casualty losses as a function of the magnitude of the earthquake. Similar to work studied by Mete and Zabinsky [12], the prepositioning of relief goods in logistics networks is addressed in Alem et al. [8]. The set of scenarios in the second stage of the model is defined as the combination of four factors, i.e., demand, supply, donation, and route damage, totaling 40 scenarios. The generation procedure of scenarios is based on a classification of the magnitudes of disasters, historical data, and the related assumptions. For example, route damage is partially proportional to the number of victims. However, studies mentioned above deal with interdependences between random factors, tend to base on single original factor, such as magnitude of earthquake, and describe other factors, e.g., number of victims, facility damage, etc. as the simple proportional relationship with the original factor. Until now, considering multiple uncertain sources of a disaster in location-inventory planning is still limited and lacks systematic method.

Moreover, it is worth noting that the studies above only focus on general relief goods. Due to the importance of human blood in disaster rescue, more and more studies focus on emergency BLN design, taking the special structure of blood supply network as well as distinctive features of blood products into account. For example, Kochan et al. [18] and Gunpinar and Centeno [19] focus on inventory decisions before disaster for a given BLN to mitigate the impacts of disasters, in which the uncertain demand and perishability of human blood are considered. Besides, facility location related decisions before disasters, mainly blood donor points and permanent blood facilities, as well as inventory decision in the BLNs in case of disasters, are also studied. For example, Fahimnia et al. [20] present a bi-objective SP model to determine the number of blood facilities in the preparedness stage and the decisions like transportation routing and blood collection in the response stage. Samani et al. [21] develop a multi-objective two-stage stochastic programming to optimize blood center location before disaster happens and assignment of donors, blood facilities, and demand zones after disaster. Shelf life of blood products and demand uncertainty are involved.

$\mathrm{SP}$ is extensively applied to the design of emergency logistics networks, including BLNs. However, in most previous studies, the stochastic scenario which is indispensable in SP is assumed to be known. Obviously, such assumption is not always realistic, especially in the case of disasters, due to their unpredictability. This motivates more researchers to utilize Robust Optimization (RO), which only requires the worst-case information instead of entire distribution, to study related problems. For example, Jabbarzadeh et al. [22] develop a robust model to investigate the design of the BLN, in which the location decisions of the permanent blood collection facilities must be made before a disaster, and adjustments in location and capacity of temporary facilities can be implemented with the consideration of the worst scenario. Khalilpourazari and Khamseh [23] extend the mathematical model presented by Jabbarzadeh et al. [22] to the bi-objective setting which aims to minimize total blood supply chain costs as well as total transportation time. Salehi et al. [24] present a robust two-stage stochastic model for the emergency BSN design, in which multiple blood products and the compatibility of the blood group are considered. Rahmani [25] proposes a robust model for the BSN design by using p-criterion technique to protect the solution against the risk of disruptions. Similar works can refer to Shishebori and Babadi [26], Zahiri et al. [27], Fereiduni and Shahanaghi [28], Ramezanian and Behboodi [29], Samani and Hosseini-Motlagh [30], and Kamyabniya et al. [31]. These works indicate that $\mathrm{RO}$ can provide robust solutions to unexpected disasters. However, RO fails to capture some valuable information contained in available data and, then, sometimes achieves too conservative solutions $[32,33]$.

To clearly demonstrate the existing BLN research under the setting of disasters, Tables 1(a) and 1(b) show the features of model, decisions, and the formulation methods of them. 


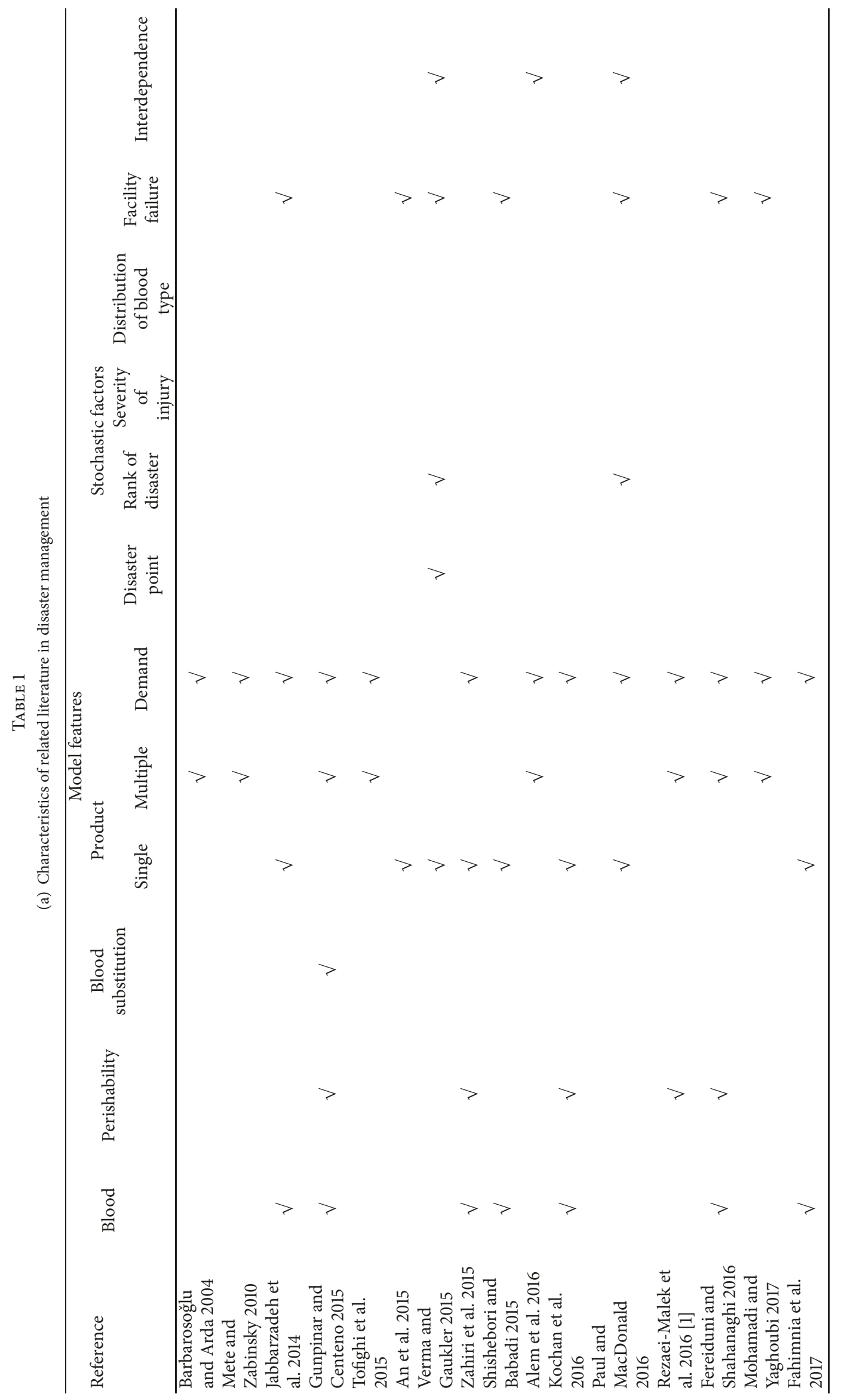




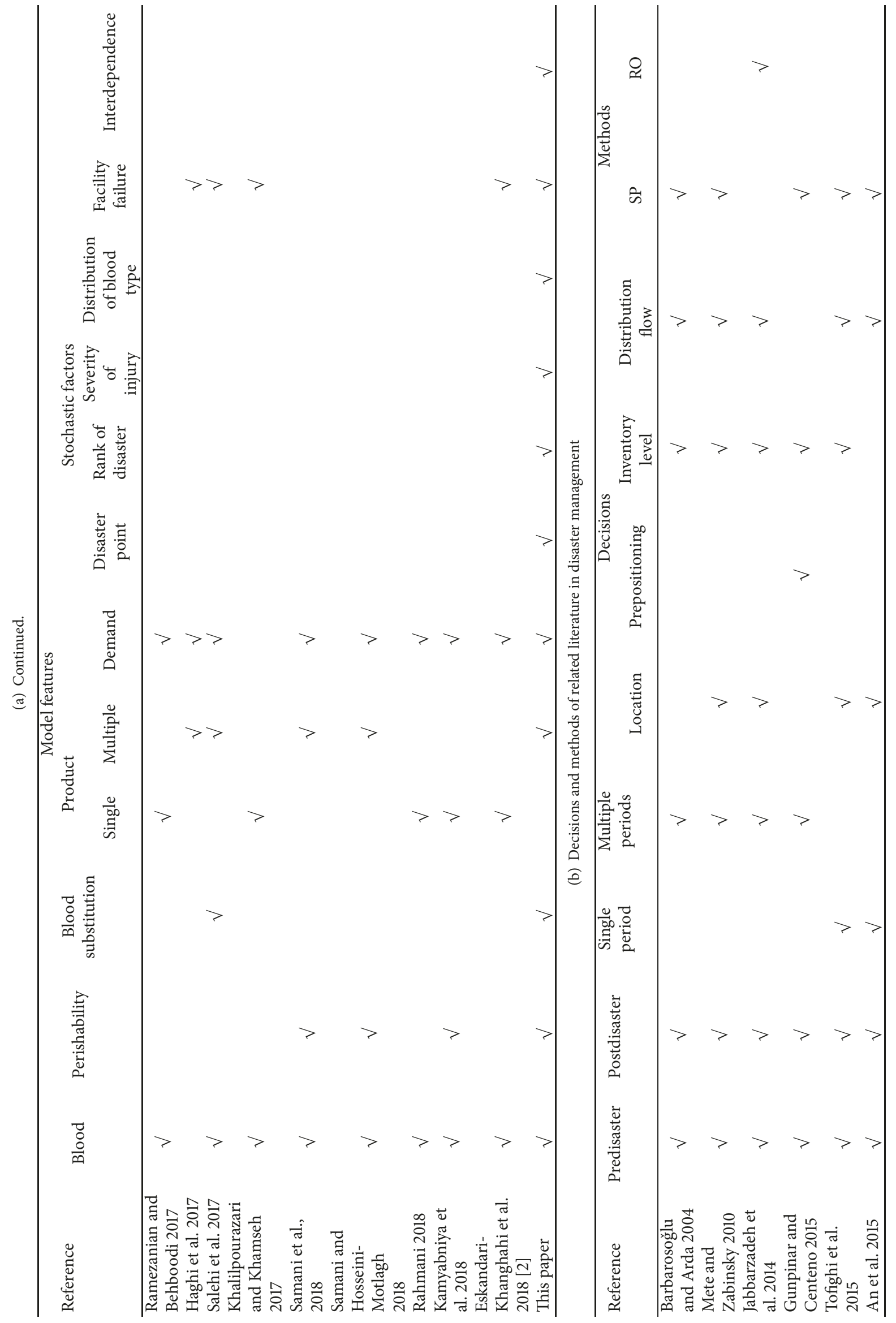




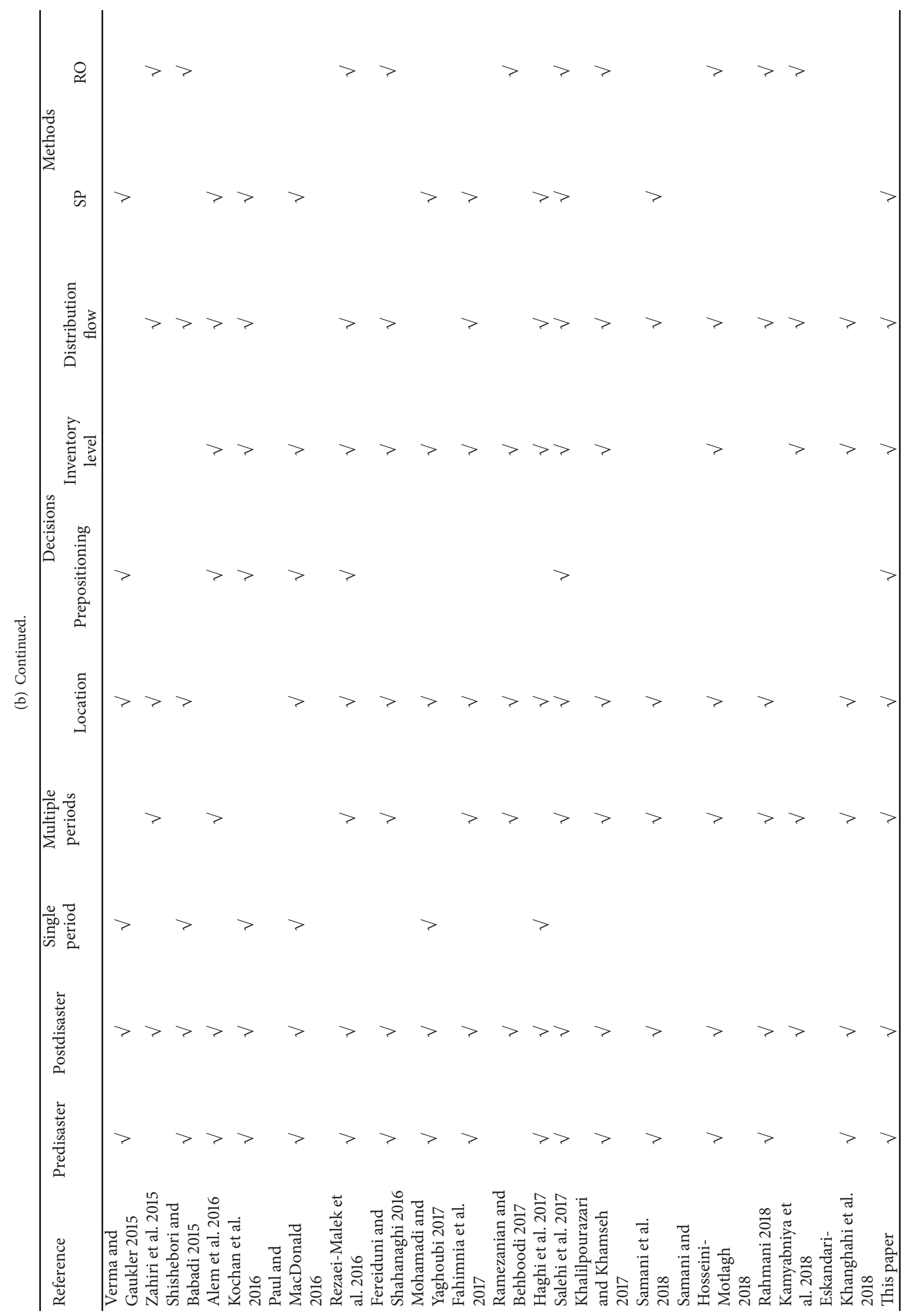


In summary, the rescue effects of BLNs are significantly influenced by the consequences of disasters, which embody in multiple aspects, including the emergency demand, facility failure, and the place where the disaster occurs. In previous studies, such consequences are treated in a relatively simple way. Specifically, most works only consider the random demand. Also, SP-based studies usually assume there is a known discrete distribution about the uncertain demand, etc. RO, which does not require a probabilistic distribution, only considers extreme scenarios and thus may lead to conservative decisions. Recently, only few BLN-related works, such as Fereiduni and Shahanaghi [28], Fahimnia et al. [20], and Salehi et al. [24], notice the importance of considering multiple disasters-related factors. However, the uncertainties considered in these works are still limited and their relationships are also ignored. Actually, if we can explore the internal relationships between multiple stochastic factors involved in disasters, it would be possible to give a precise description of the consequences of disasters and then, facilitate the application of sophisticated mathematical programming methodologies, such as SP. Thus, what we need is a quantitative method which can systematically formulate the relationship between multiple random factors in disasters.

In recent years, BNs have become a popular method for extracting knowledge from data in complex and uncertain systems, which encode the joint probability distribution of a set of random variables by making conditional dependence assumptions [34]. As suggested by Galindo and Batta [9], the Bayesian approach could be suitable for incorporating additional observations into stochastic operations management. Till now, BN has been applied to evaluate the occurrence probability and consequence of the accident in different areas, such as the reliability of semiconductor manufacturing [35], mine water inrush [36], navigation safety [37], gas explosion [38], and tropical cyclones [39]. But, its applications in operation research are still rare, not to mention BLNs.

These works indicate that BNs can be used to describe complicated disaster scenarios and improve the quality of decisions. However, it is worth noting that different types of disasters involve distinctive stochastic factors and their relationships are also diverse. Fortunately, for some common natural disasters, such as earthquakes and hurricanes, there are lots of studies in the context of disasters research which can provide solid foundation for our BN modelling. Moreover, with the consideration of more stochastic factors, we need to consider more decisions of BLNs, which means a dedicated model is required. Thus, in the next section, we describe the problem we studied, give the corresponding mathematical formulation and then, propose our BN for the case of earthquake.

\section{Problem Definition and Model Formulation}

3.1. Problem Statement. Consider a region where a government plans to launch a central blood bank to satisfy both the daily demand and the uncertain demand caused by possible future disasters. Hence, the location-inventory problem is proposed in a three-layer BLN composed by donor points, a central blood bank, and hospitals, taking uncertain disasters and the characteristics of human blood into account. After the blood bank is located, the donor points collect blood from donors and provide blood to the prespecified blood bank, which is responsible for distributing blood to hospitals. The operational stage is divided into multiple periods (time interval of each period is $T$ ), some of which suffer suddenly-occurring disasters. The activities mentioned above repeatedly happen in each daily period. When a disaster occurs in a period, the casualties are transferred from the disaster point to the rescue hospital which is the closest available one. Then, the rescue hospital and the prespecified blood bank should satisfy the emergency demand at the firsttime treatment by their prepositioning inventory without interruption. Obviously, this is critical to the medical relief for the victims. The objective is to minimize the total cost of the BLN while ensuring that the blood demands are met under both daily and disaster situations in a given planning horizon.

The problem consists of four different decisions: (1) the central blood bank location; (2) inventory level in the prespecified blood bank and each hospital at the beginning of each operational period; (3) the distribution flow from donor points to the prespecified blood bank at each operational period; and (4) the distribution flow from the prespecified blood bank to each hospital at each operational period. The decisions are made in two stages. Taking the BLN composed by three donor points, two central blood bank candidates and two hospitals as the example, the planning stage includes the strategic decisions on the selection of a central blood bank from two candidates and the inventory level of both the selected blood bank and each hospital. Given these strategic decisions, the activities in one period of the operational stage under the daily or disaster setting are given respectively in Figure 1.

The daily operations of the whole BLN are displayed in Figure 1(1). In each period, the donor points collect the blood as the request of the central blood bank, and then the central blood bank implements blood test, inspection, etc., and replenishes each hospital by using its inventory at the same time.

More specifically, the inventory status of each facility in Figure 1(1) can be further illustrated by Figure 2 which shows the change of the inventory level of each corresponding facility.

As shown in the right part in Figure 2, the shipments from donor points to the blood bank and from the blood bank to each hospital should begin at the beginning of each period. Actual arrival time is dependent on the corresponding transportation distance. The in-transit inventory is a part of the inventory level of the corresponding receiver which also accounts for a portion of its holding cost. The right-upper part of Figure 2 shows that three donor points collect the different quantity of blood in each period which composes the total blood supply. Moreover, as shown in the right-middle part of Figure 2, the inventory of blood in the prespecified blood bank consists of the daily inventory and the emergency stock. The former is used to meet the daily deterministic demand from hospitals at each daily period, 


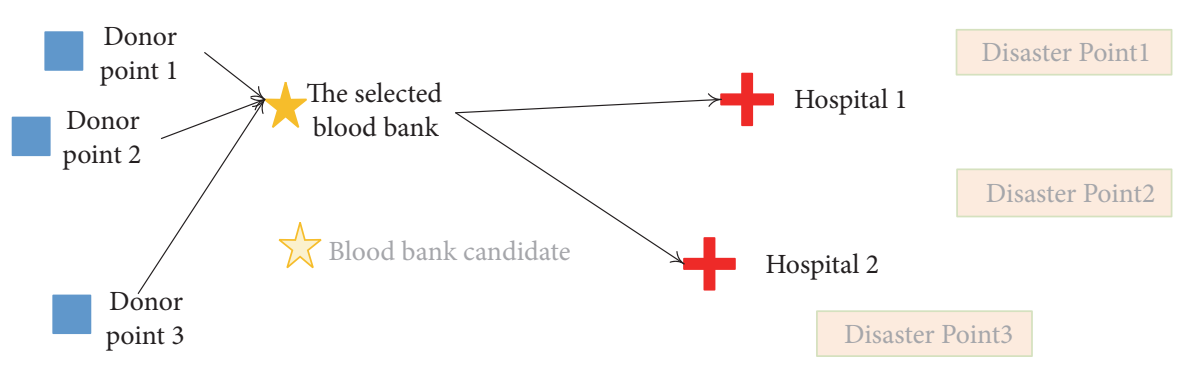

(1) A daily period

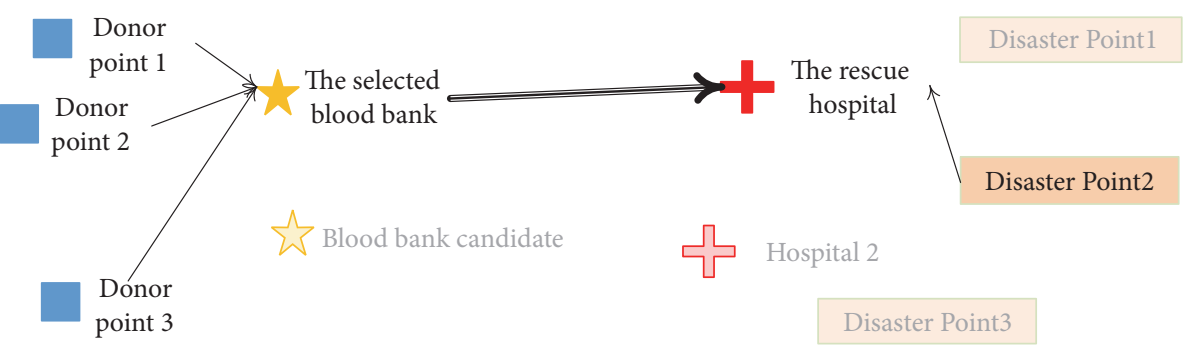

(2) A disaster operational period

FIgURE 1: The illustration of activities in one period of the operational stage.

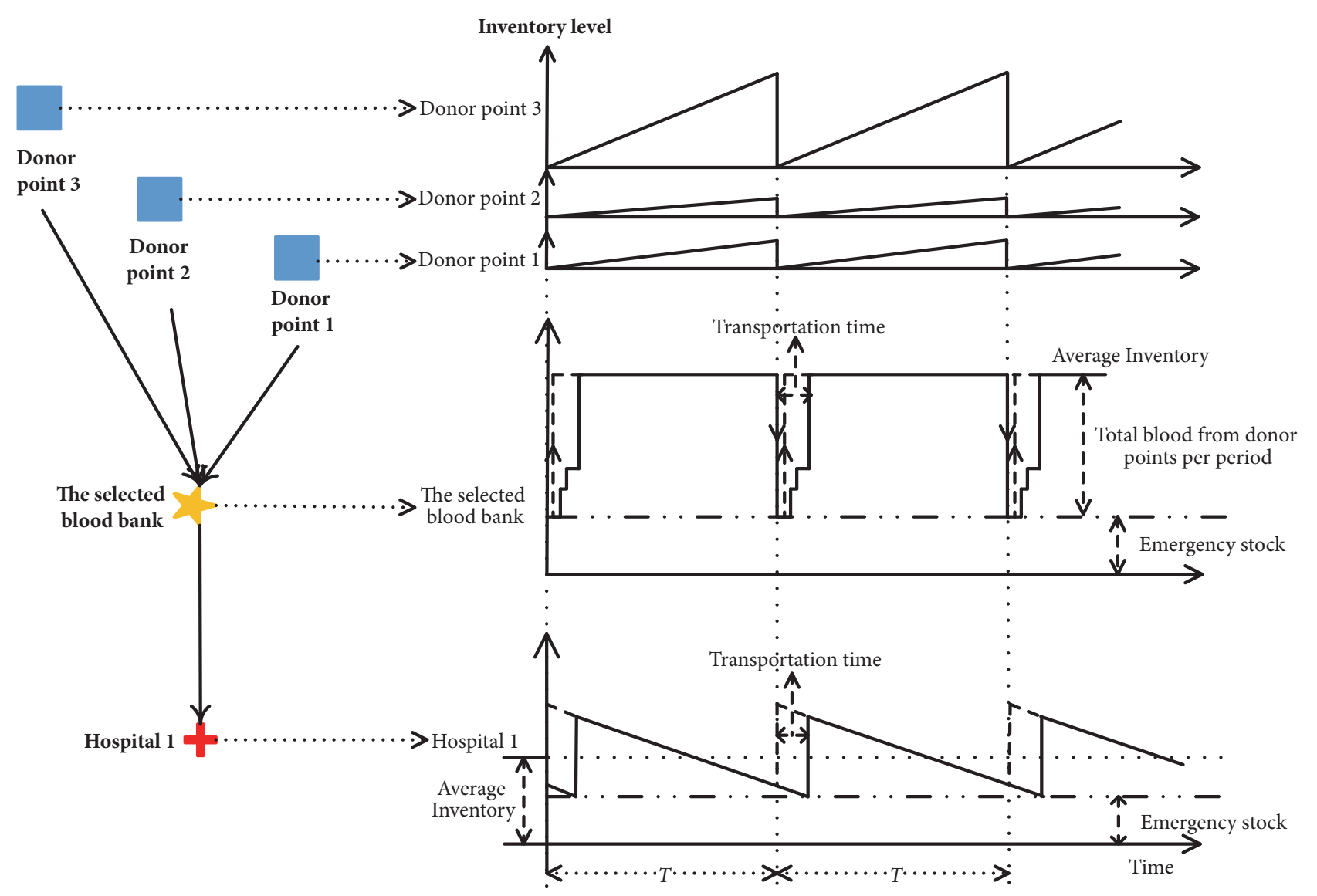

FIgURE 2: The status of inventory level in three-layers BLN in the operational stage. 
and the latter is prepositioned to respond to the disaster demand. Both of them are decided at the planning stage. The total inventory level should be the sum of its physical inventory (denoted as the solid staircase lines) and the intransit inventory (denoted as the dashed arrows). Finally, the inventory in each hospital (see the right-lower part of Figure 2) also includes the daily inventory and the emergency stock, in which the latter is prepositioned for the case that the casualties arrive at the hospital earlier than the delivery of blood from the prespecified blood bank. Similarly, the holding cost of each hospital is calculated based on the sum of its in-transit inventory (denoted as the dashed lines) from the prespecified blood bank and its physical inventory (denoted as solid lines).

Figure 1(2) illustrates the case that the disaster happens in some potential disaster points (e.g., disaster point 2), and then the casualties would be sent to the closest available hospital, namely, the rescue hospital (e.g., hospital 1) in some periods. Thus, the rescue hospital needs to use its inventory to satisfy the emergency demand caused by the disaster at first. To ensure the continuous transfusion, the prespecified blood bank also should have the ability to use its prepositioning inventory to replenish the blood to the rescue hospital before its inventory runs out. The continuous transfusion time in the first-time treatment is $\Delta T$, and the blood transfusion quantity is directly influenced by the consequences of the disaster, such as the number of the injured and the severity of the injury. These uncertain factors and the stochastic disaster points, as well as the availability of hospitals, would be directly or indirectly considered in the proposed model.

In summary, the BLN itself should have the ability to satisfy the first-time blood demand. The consumed inventory in the BLN can be recovered before the next period. Regarding the blood demand of subsequent treatments on the injured, it also can be satisfied by other neighbor regional BLNs or extra blood donations, which is not discussed in our study.

The main assumptions of the above problem are listed as follows:

(i) A fixed construction cost for each blood bank candidate is available.

(ii) The holding cost of in-transit inventory from each donor point to the prespecified blood bank and the prespecified blood bank to each hospital will be included in the inventory cost of the blood bank and corresponding hospital, respectively.

(iii) The inventory holding cost is proportional to the inventory duration and quantity.

(iv) The transportation cost is proportional to the distance and distribution flow.

(v) In each period, at most one disaster occurs.

(vi) At least one hospital is available during the disaster period, and the unavailable hospital can recover in the next period.

The last two assumptions are reasonable in our study. First, the disasters that require emergency blood rescue usually would not occur frequently. Moreover, a hospital may be unavailable because of surrounding road damage or the damage to the hospital itself in a disaster. However, even all hospitals are unavailable, in order to implement the relief, the government would restore the most slightly damaged one or build a temporary rescue facility nearby some hospital as soon as possible. Hence, it is reasonable to assume there is at least one available hospital. Furthermore, some hospitals that are seriously damaged may not be restored soon; however, the daily demand in the area covered by the damaged hospital still exists and may be guided to a nearby substituting facility in a short time. Thus, the similar daily operational activities still exist. Therefore, we give the last assumption.

\subsection{Model Formulation}

(a) Model Formulation and Explanation. As it was previously pointed out, the decisions are made in two stages. The planning stage includes the decisions on the location of a central blood bank and the inventory levels in both the prespecified blood bank and hospitals. A binary location decision variable $x_{i}$ equals to 1 if the central blood bank candidate $i$ is selected, or 0 otherwise. Moreover, $C_{i a b}$ represents the maximum inventory level of blood products $a$ with type $b$ in the candidate $i . s s_{i a b}$ and $s_{h a b}$ are emergency stock levels in the candidate $i$ and the hospital $h$, respectively. The operational stage includes the distribution flow in both each daily period and disaster period. In each daily period, the distribution flow of blood products $a$ with type $b$ from the donor point $k$ to the candidate $i$ is represented as $g_{\text {kiab }} . w_{\text {ihab }}$ and $w^{\prime}{ }_{i h a b b^{\prime}}$ are the distribution flows from the candidate $i$ to the hospital $h$. The latter is the quantity of blood product $a$ with type $b^{\prime}$ used to substitute type $b$. When a disaster occurs, $g_{k i a b}^{\prime s}$ is used to represent the distribution flow of blood products $a$ with type $b$ from the donor point $k$ to the candidate $i$ under scenario $s$. Let $y_{i h a b}^{s}$ and $y_{i h a b b^{\prime}}^{\prime s}$ be the distribution flow from the candidate $i$ to the rescue hospital $h$ with the consideration of blood substitution under scenario $s$.

Sets, parameters, and variables used are given in Tables $2-4$. The mutual substitution relationship, i.e., variable $q_{b b^{\prime}}$, of different blood types in set BLN, is given in Table 5 [7].

The objective of the two-stage multiperiod SP model aims at minimizing the total cost of the BLN in both the planning stage and operational stage, which can be divided into three terms: (1) the construction cost in the planning stage, (2) the operational cost of the daily period, and (3) the operational cost of the disaster period in the operational stage.

(1) The Construction Cost in the Planning Stage. It can be formulated as formula (1):

$$
\sum_{i \in I} c_{i} \cdot x_{i}
$$

(2) The Operational Cost of the Daily Period in the Operational Stage. The daily operational cost includes the inventory holding cost of the prespecified blood bank, the inventory holding cost of hospitals, the transportation cost of distribution flow from donor points to the prespecified blood bank, and the 
TABLE 2: Sets and indices.

\begin{tabular}{lc}
\hline Symbol & Description \\
\hline$K$ & Set of donor points \\
$I$ & Set of blood bank candidates \\
$H$ & Set of hospitals \\
$A$ & $\begin{array}{c}\text { Set of blood products (plasma, } \\
\text { platelets, et al.) }\end{array}$ \\
$B L$ & Set of blood types (A, B, AB, O, \\
$S_{1}$ & et al.) \\
$S$ & Set of disaster scenarios \\
& Set of whole scenarios (union of \\
$k$ & $S_{1}$ and the scenario without a \\
$i$ & disaster) \\
$h$ & Indices to donor points, and $k \in K$ \\
$a$ & Indices to blood bank candidates, \\
& and $i \in I$ \\
$b$ & Indices to hospitals, and $h \in H$ \\
$s$ & Indices to blood products, and \\
& Indices to blood types, and $b \in B L$ \\
\hline & Indices to scenarios, and $s \in S$ \\
\hline
\end{tabular}

transportation cost of distribution flow from the prespecified blood bank to hospitals. Thus, the operational cost in one period can be calculated as

$$
\begin{aligned}
& T \cdot \sum_{i \in I}\left[r_{i} \cdot\left(\sum_{a \in A} \sum_{b \in B L} s s_{i a b}+\sum_{k \in K} \sum_{a \in A} \sum_{b \in B L} g_{k i a b}\right)\right]+T \\
& \cdot \sum_{h \in H}\left[u_{h}\right. \\
& \left.\cdot \sum_{a \in A} \sum_{b \in B L}\left(\sum_{i \in I} \frac{x_{i} \cdot\left(T+t_{i h}\right) \cdot d_{h a b}}{2}+s_{h a b}\right)\right]+e \cdot v \\
& \cdot \sum_{i \in I} \sum_{k \in K}\left(t_{k i} \cdot \sum_{a \in A b \in B L} \sum_{k i a b}\right)+e \cdot v \cdot \sum_{i \in I} \sum_{h \in H}\left(t_{i h}\right. \\
& \left.\cdot \sum_{a \in A} \sum_{b \in B L}\left(w_{i h a b}+\sum_{b^{\prime} \in B L, b^{\prime} \neq b} w_{\text {ihabb }}^{\prime}\right)\right)
\end{aligned}
$$

in which, $\sum_{a \in A} \sum_{b \in B L} s s_{i a b}+\sum_{k \in K} \sum_{a \in A} \sum_{b \in B L} g_{k i a b}$ is the inventory level of blood bank candidate $i$ which involves the inventory on-hand and the corresponding in-transit inventory. When candidate $i$ is not selected, this level would be set to zero by the constraints later. Similarly, $\sum_{a \in A} \sum_{b \in B L}\left(\sum_{i \in I}\left(x_{i}\right.\right.$. $\left.\left.\left(T+t_{\text {ih }}\right) \cdot d_{\text {hab }} / 2\right)+s_{\text {hab }}\right)$ is the inventory level of hospital $h$ in which $\sum_{i \in I}\left(x_{i} \cdot\left(T+t_{i h}\right) \cdot d_{\text {hab }} / 2\right)$ is the average cycle inventory per $T$.

(3) The Operational Cost of the Disaster Period in the Operational Stage. In the period which suffers a disaster, the operational cost includes four items, in which the first two items are the same as these in formula (2). The third one is the transportation cost from the donor points to the prespecified blood bank in the disaster period. Then the last one is the transportation cost caused by the daily and emergency demand of the rescue hospital as well as the daily demand of other available hospitals. Hence, the costs in a disaster period under one scenario $s$ can be represented as follows:

$$
\begin{aligned}
& T \cdot \sum_{i \in I}\left[r_{i} \cdot\left(\sum_{a \in A} \sum_{b \in B L} s s_{i a b}+\sum_{k \in K} \sum_{a \in A} \sum_{b \in B L} g_{k i a b}\right)\right]+T \\
& \cdot \sum_{h \in H}\left[u_{h} \cdot \sum_{a \in A} \sum_{b \in B L}\left(\sum_{i \in I} \frac{x_{i} \cdot\left(T+t_{i h}\right) \cdot d_{h a b}}{2}+s_{h a b}\right)\right] \\
& +e \cdot v \cdot \sum_{i \in I} \sum_{k \in K}\left(t_{k i} \cdot \sum_{a \in A} \sum_{b \in B L} g_{k i a b}^{\prime s}\right)+e \cdot v \\
& \cdot \sum_{i \in I} \sum_{h \in H}\left(t_{i h} \cdot L_{h}^{s}\right. \\
& \left.\cdot \sum_{a \in A} \sum_{b \in B L}\left(y_{\text {ihab }}^{s}+\sum_{b^{\prime} \in B L, b^{\prime} \neq b} y_{\text {ihabb }}^{\prime s}\right)\right)
\end{aligned}
$$

In our study, the scenario set in each operational period is denoted as $S$, which contains the disaster scenario $s\left(\in S_{1}\right)$ with the probability $p^{s}$ and the normal scenario $s_{0}\left(\in S-S_{1}\right)$ with the probability $p^{s_{0}}$. Thus, we have $p^{s_{0}}+\sum_{s \in S_{1}} p^{s}=1$. The disaster scenarios vary with the different disaster points, the ranks of disasters, the unavailability of each hospital, etc.

Notice that the first two cost items in formula (2) (or formula (3)) always exist in the operational stage, while the last two in formula (2) and formula (3) are associated with the corresponding probability. Then, the final objective function of the two-stage multiperiod SP model is formulated as

$$
\begin{aligned}
& \min Z=\sum_{i \in I} c_{i} \cdot x_{i}+\Phi \cdot\left\{T \cdot \sum _ { i \in I } \left[r _ { i } \cdot \left(\sum_{a \in A} \sum_{b \in E L} s s_{a b b}\right.\right.\right. \\
& \left.\left.+\sum_{k \in K} \sum_{a \in A} \sum_{b \in B L} g_{k i a b}\right)\right]+T \cdot \sum_{h \in H}\left[u_{h}\right. \\
& \left.\left.\sum_{a \in A} \sum_{b \in E L}\left(\sum_{i \in I} \frac{x_{i} \cdot\left(T+t_{i h}\right) \cdot d_{h a b}}{2}+s_{\text {hab }}\right)\right]\right\}+\Phi \\
& \cdot\left\{p ^ { s _ { 0 } } \cdot e \cdot v \cdot \left[\sum _ { i \in \epsilon } \sum _ { h \in H } \left(t_{i h}\right.\right.\right.
\end{aligned}
$$

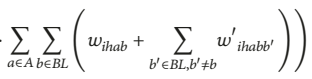

$$
\begin{aligned}
& \left.+\sum_{i \in \in \in k \in K}\left(t_{k i} \cdot \sum_{a \in A \in \in B L} \sum_{k i a b}\right)\right]+\sum_{s \in S_{1}}\left[p^{s} \cdot e \cdot v\right.
\end{aligned}
$$

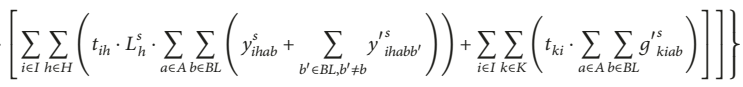

Moreover, the constraints include the following three groups.

(1) The Constraints in the Planning Stage. In the planning stage, we would select one central blood bank among several candidates and make decisions on the maximum inventory of 
TABLE 3: Model parameters.

\begin{tabular}{|c|c|c|}
\hline & Symbol & Description \\
\hline \multirow{16}{*}{ Deterministic parameters } & $\Phi$ & The number of periods in planning horizon \\
\hline & $T$ & Time interval of each period \\
\hline & $e$ & Unit transportation fee $(\mathrm{RMB} / \mathrm{km} \bullet \mathrm{U}, \mathrm{U}$ is the abbreviation of blood Units. 1 Unit equals $200 \mathrm{ml})$ \\
\hline & $v$ & The velocity of transportation $(\mathrm{km} / \mathrm{h})$ \\
\hline & $\alpha$ & Demand service level (confidence level) \\
\hline & $r_{i}$ & Inventory holding cost per unit of blood bank $i(\mathrm{RMB} / \mathrm{U} \bullet \mathrm{h})$ \\
\hline & $c_{i}$ & Fixed construction cost of blood bank $i$ (RMB) \\
\hline & $u_{h}$ & Inventory holding cost per unit of hospital $h(\mathrm{RMB} / \mathrm{U} \bullet \mathrm{h})$ \\
\hline & $t_{k i}$ & Transportation time from donor point $k$ to blood bank $i(\mathrm{~h})$ \\
\hline & $t_{i h}$ & Transportation time from blood bank $i$ to hospital $h(\mathrm{~h})$ \\
\hline & $d i s_{n h}$ & Distance from potential disaster point $n$ to hospital $h .(\mathrm{km})$ \\
\hline & $l_{a}$ & Lifespan of blood product $a(\mathrm{~h})$ \\
\hline & $f_{k a b}$ & Supply capacity of blood product $a$ with type $b$ in donor point $k(\mathrm{U})$ \\
\hline & $d_{\text {hab }}$ & Daily demand for blood product $a$ with type $b$ per hour in hospital $h(\mathrm{U} / \mathrm{h})$ \\
\hline & $q_{b b^{\prime}}$ & 1 if blood type $b^{\prime}$ is able to substitute type $b, 0$ otherwise. \\
\hline & $\Delta T$ & The continuous blood transfusion time in the first-time treatment $(\mathrm{h})$ \\
\hline \multirow{5}{*}{ Stochastic parameters } & $p^{s}$ & Probability of scenario $s(\in S)$ \\
\hline & $t_{h}^{s}$ & Transportation time from the actual disaster point to hospital $h$ under scenario $s(\mathrm{~h})$ \\
\hline & $L_{h}^{s}$ & 1 if hospital $h$ is available to the injured under scenario $s, 0$ otherwise. \\
\hline & $z_{h}^{s}$ & 1 if the injured are sent to hospital $h$ under scenario $s, 0$ otherwise. \\
\hline & $d_{a b}^{s}$ & Emergency demand for blood product $a$ with type $b$ under scenario $s(\mathrm{U} / \mathrm{h})$ \\
\hline
\end{tabular}

TABLE 4: Decision variables.

\begin{tabular}{|c|c|c|}
\hline & Symbol & Description \\
\hline \multirow{4}{*}{$\begin{array}{l}\text { The } \\
\text { planning } \\
\text { stage }\end{array}$} & $x_{i}$ & 1 if blood bank candidate $i$ is chosen, 0 otherwise. \\
\hline & $C_{i a b}$ & $\begin{array}{l}\text { Maximum inventory of blood product } a \text { with type } b \text { in blood bank } \\
\text { candidate } i(\mathrm{U})\end{array}$ \\
\hline & $s s_{i a b}$ & $\begin{array}{c}\text { Emergency stock of blood product } a \text { with type } b \text { in blood bank } \\
\text { candidate } i(\mathrm{U})\end{array}$ \\
\hline & $s_{\text {hab }}$ & Emergency stock of blood product $a$ with type $b$ in hospital $h(\mathrm{U})$ \\
\hline \multirow{6}{*}{$\begin{array}{l}\text { The } \\
\text { operational } \\
\text { stage }\end{array}$} & $g_{\text {kiab }}$ & $\begin{array}{c}\text { Supply quantity of blood product } a \text { with type } b \text { from donor point } k \text { to } \\
\text { blood bank candidate } i \text { per period (U) }\end{array}$ \\
\hline & $w_{\text {ihab }}$ & $\begin{array}{l}\text { Distribution flow of blood product } a \text { with type } b \text { from blood bank } i \text { to } \\
\text { hospital } h \text { per period (U) }\end{array}$ \\
\hline & $w_{i h a b b^{\prime}}^{\prime}$ & $\begin{array}{l}\text { Distribution flow of blood product } a \text { with type } b^{\prime} \text { used to substitute } \\
\text { type } b \text { from blood bank } i \text { to hospital } h \text { per period (U) }\end{array}$ \\
\hline & $g_{k i a b}^{\prime s}$ & $\begin{array}{l}\text { Supply quantity of blood product } a \text { with type } b \text { from donor point } k \text { to } \\
\text { blood bank candidate } i \text { under scenario } s(\mathrm{U})\end{array}$ \\
\hline & $y_{\text {ihab }}^{s}$ & $\begin{array}{l}\text { Distribution flow of blood product } a \text { with type } b \text { from blood bank } i \text { to } \\
\text { hospital } h \text { under scenario } s(\mathrm{U})\end{array}$ \\
\hline & $y^{\prime s}{ }_{i h a b b^{\prime}}$ & $\begin{array}{l}\text { Distribution flow of blood product } a \text { with type } b^{\prime} \text { used to substitute } \\
\text { type } b \text { from blood bank } i \text { to hospital } h \text { under scenario } s(\mathrm{U})\end{array}$ \\
\hline
\end{tabular}

each blood product with different blood type. The constraints are represented as follows:

$$
\begin{gathered}
\sum_{i \in I} x_{i}=1 \\
\max _{k \in K ; i \in I ; h \in H}\left(t_{k i}+t_{i h}\right) \cdot x_{i} \leq \min l_{a}, \quad a \in A \\
\sum_{a \in A} \sum_{b \in B L} C_{i a b} \leq M M \cdot x_{i}, \quad i \in I
\end{gathered}
$$

$$
\sum_{k \in K} g_{k i a b}+s s_{i a b} \leq C_{i a b},
$$

$$
i \in I ; a \in A ; b \in B L
$$

$$
x_{i} \in\{0,1\}, \quad i \in I
$$$$
C_{i a b}, s s_{i a b}, s_{h a b}, \geq 0 \text {, }
$$

$i \in I ; h \in H ; a \in A ; b \in B L$ 
TABle 5: The mutual substitution among common blood types (set $B L$ ).

\begin{tabular}{|c|c|c|c|c|c|c|c|c|c|}
\hline \multirow{2}{*}{\multicolumn{2}{|c|}{$\begin{array}{l}q_{b b^{\prime}} \\
b, b^{\prime}(\in B L)\end{array}$}} & \multicolumn{8}{|c|}{$b$} \\
\hline & & $\mathrm{O}+$ & $\mathrm{O}_{-}$ & $\mathrm{A}+$ & A- & $\mathrm{B}+$ & B- & $\mathrm{AB}+$ & $\mathrm{AB}-$ \\
\hline \multirow{8}{*}{$b^{\prime}$} & $\mathrm{O}+$ & 1 & 0 & 1 & 0 & 1 & 0 & 1 & 0 \\
\hline & $\mathrm{O}_{-}$ & 1 & 1 & 1 & 1 & 1 & 1 & 1 & 1 \\
\hline & $\mathrm{A}+$ & 0 & 0 & 1 & 0 & 0 & 0 & 1 & 0 \\
\hline & A- & 0 & 0 & 1 & 1 & 0 & 0 & 1 & 1 \\
\hline & $\mathrm{B}+$ & 0 & 0 & 0 & 0 & 1 & 0 & 1 & 0 \\
\hline & B- & 0 & 0 & 0 & 0 & 1 & 1 & 1 & 1 \\
\hline & $\mathrm{AB}+$ & 0 & 0 & 0 & 0 & 0 & 0 & 1 & 0 \\
\hline & AB- & 0 & 0 & 0 & 0 & 0 & 0 & 1 & 1 \\
\hline
\end{tabular}

Constraint (5) ensures only one central blood bank would be chosen from all blood bank candidates in a region. Constraints (6) require that the time of transportation from any donor point to any hospital should be within the shortest lifespan of corresponding blood product. $M M$ in (7) is a number big enough. Thus, inequalities (7) ensure that the corresponding maximum inventory of unselected blood bank candidate is zero. Constraints (8) enforce that the total quantity of blood provided by donor points within each $T$ and the emergency stock is less than the maximum inventory in corresponding blood bank. Constraints (9) specify the domains of the decision variables.

(2) The Constraints of Daily Periods in the Operational Stage. In the daily operational period, the distribution flow should not be more than the quantity supplied by donor points and should meet the demand of all hospitals. The constraints are shown as follows:

$$
\begin{aligned}
& 0 \leq \sum_{i \in I} g_{k i a b} \leq f_{k a b}, \quad k \in K ; a \in A ; b \in B L \\
& \sum_{h \in H}\left(w_{i h a b}+\sum_{b^{\prime} \in B L, b^{\prime} \neq b} q_{b b^{\prime}} \cdot w_{i h a b^{\prime} b}^{\prime}\right) \leq \sum_{k \in K} g_{k i a b}, \\
& \qquad i \in I ; a \in A ; b \in B L \\
& x_{i} \cdot\left(T+t_{i h}\right) \cdot d_{h a b} \leq w_{i h a b}+\sum_{b^{\prime} \in B L, b^{\prime} \neq b} q_{b b^{\prime}} \cdot w_{i h a b b^{\prime}}^{\prime}, \\
& \qquad i \in I ; h \in H ; a \in A ; b \in B L \\
& x_{i} \in\{0,1\}, \quad i \in I \\
& g_{k i a b}, w_{\text {ihab }}, w_{\text {ihabb }}^{\prime} \geq 0,
\end{aligned}
$$$$
i \in I ; h \in H ; a \in A ; b, b^{\prime} \in B L
$$

Constraints (10) express the maximum supply quantity in each donor point. Constraints (11) specify that the quantity of blood supplied to hospitals should be less than the quantity provided by all donor points within each $T$. Constraints (12) ensure that the blood delivery to hospitals should meet the demand during the sum of $T$ and the transportation time with the consideration of mutual substitution among blood types. Constraints (13) specify the domains of the decision variables.

(3) The Constraints of Disaster Periods in the Operational Stage. When a disaster happens, the injured are sent to the rescue hospital which should meet both the daily and emergency blood demand by its inventory at the first time. Besides, the subsequent blood should be supplied by the central blood bank to ensure continuous transfusion. Other available hospitals still need to satisfy their own daily demand. Thus, the constraints are formulated as

$$
\begin{array}{r}
\sum_{h \in H} L_{h}^{s} \cdot\left(y_{i h a b}^{s}+\sum_{b^{\prime} \in B L, b^{\prime} \neq b} q_{b b^{\prime}} \cdot y^{\prime s}{ }_{i h a b^{\prime} b}^{s}\right) \\
s \in S_{1} ; i \in I ; a \in A ; b \in B L
\end{array}
$$

$$
\begin{aligned}
& \sum_{h \in H} L_{h}^{s} \cdot\left(y_{i h a b}^{s}+\sum_{b^{\prime} \in B L, b^{\prime} \neq b} q_{b b^{\prime}} \cdot y_{i h a b^{\prime} b}^{\prime s}\right) \leq \sum_{k \in K} g_{k i a b} \\
& \quad+s s_{i a b}, \quad s \in S_{1} ; \quad i \in I ; a \in A ; b \in B L \\
& \frac{s_{h a b}}{d_{a b}^{s}}+t_{h}^{s} \geq x_{i} \cdot z_{h}^{s} \cdot t_{i h},
\end{aligned}
$$$$
s \in S_{1} ; h \in H ; i \in I ; a \in A ; b \in B L
$$

$$
\begin{aligned}
& y_{\text {ihab }}^{s}+\sum_{b^{\prime} \in B L, b^{\prime} \neq b} q_{b b^{\prime}} \cdot y_{i h a b b^{\prime}}^{\prime s} \geq x_{i} \cdot\left(T+t_{i h}\right) \cdot d_{h a b} \\
& \cdot L_{h}^{s}, \quad s \in S_{1} ; i \in I ; h \in H ; a \in A ; b \in B L \\
& P\left\{\sum _ { i \in I } \left[\left(y_{i h a b}^{s}+\sum_{b^{\prime} \in B L, b^{\prime} \neq b} q_{b b^{\prime}} \cdot y_{i h a b b^{\prime}}^{\prime s}-x_{i} \cdot L_{h}^{s} \cdot(T\right.\right.\right. \\
& \left.\left.\left.\left.+t_{i h}\right) \cdot d_{h a b}\right)\right]+s_{h a b} \geq \Delta T \cdot d_{a b}^{s}\right\} \geq z_{h}^{s} \cdot \alpha, \\
& s \in S_{1} ; h \in H ; a \in A ; b \in B L
\end{aligned}
$$


TABLE 6: Variables (nodes) and states in the BN.

\begin{tabular}{|c|c|c|c|}
\hline \multicolumn{2}{|c|}{ Variables (Nodes) } & \multirow{2}{*}{ States } & \multirow{2}{*}{ Description } \\
\hline Set & Name & & \\
\hline$C$ & $\begin{array}{l}\text { Potential } \\
\text { epicenters }\end{array}$ & $\begin{array}{c}\text { Point } 1 \\
\ldots \\
\text { Point } N\end{array}$ & $\begin{array}{l}\text { Consider several potential epicenters and each with corresponding earthquake } \\
\text { occurrence probabilities. }\end{array}$ \\
\hline$F$ & $\begin{array}{l}\text { Level of } \\
\text { earthquake }\end{array}$ & $\begin{array}{c}\text { Level } 1 \\
\ldots \\
\text { Level } M\end{array}$ & $\begin{array}{l}\text { Earthquake can be divided into different levels, and each of them has the } \\
\text { corresponding probabilities. }\end{array}$ \\
\hline G & $\begin{array}{l}\text { Level of } \\
\text { injury }\end{array}$ & $\begin{array}{c}\text { Seriously } \\
\text { Slight }\end{array}$ & $\begin{array}{l}\text { The level of injury of each injured person is subject to the two-point distribution. } \\
\text { Each level of injury has corresponding probability and demand of blood. }\end{array}$ \\
\hline$D$ & $\begin{array}{l}\text { Distribution } \\
\text { of blood type }\end{array}$ & $\begin{array}{c}\text { Distribution } 1 \\
\ldots \\
\text { Distribution } J\end{array}$ & The distribution of different blood types in the injured may be uncertain. \\
\hline$L$ & $\begin{array}{l}\text { States of } \\
\text { hospitals }\end{array}$ & $\begin{array}{l}\text { Unavailable } \\
\text { Available }\end{array}$ & $\begin{array}{l}\text { Use unavailable probability, which is affected by the random events in } C \text { and } F \text {, to } \\
\text { represent the state of hospitals. }\end{array}$ \\
\hline
\end{tabular}

$$
\begin{aligned}
& \sum_{s \in S_{1}}\left[\sum_{k \in K} \sum_{a \in A} \sum_{b \in B L} g_{k i a b}^{\prime s}\right. \\
& \left.\quad+\sum_{h \in H} \sum_{a \in A} \sum_{b \in B L}\left(y_{i h a b}^{s}+\sum_{b^{\prime} \in B L, b^{\prime} \neq b} y^{\prime s}{ }_{i h a b b^{\prime}}\right)\right] \\
& \left.\leq M M \cdot x_{i}, \quad i \in I \sum_{b^{\prime} \in B L, b^{\prime} \neq b} y^{\prime s}{ }_{i h a b b^{\prime}}\right) \leq M M \cdot L_{h}^{s}, \\
& \sum_{i \in I} \sum_{a \in A} \sum_{b \in B L}\left(y_{i h a b}^{s}+\sum_{i} s \in S_{1} ; h \in H\right. \\
& x_{i} \in\{0,1\}, \quad i \in I \\
& g_{k i a b}, s s_{i a b}, s_{h a b}, g_{k i a b}^{\prime s}, y_{i h a b}^{s}, y_{i h a b b^{\prime}}^{\prime s} \geq 0, \\
& s \in S_{1} ; i \in I ; h \in H ; a \in A ; b, b^{\prime} \in B L
\end{aligned}
$$

Constraints (14) represent the quantity of blood delivered from donor points to the prespecified blood bank in disaster period. Constraints (15) show the capacity of the prespecified blood bank should be able to deal with blood demand under the disaster scenario $s$. Constraints (16) guarantee that the blood replenished by the prespecified blood bank should be sent to the rescue hospital before its inventory runs out. The rescue hospital can be determined by formulas (22):

$$
\begin{aligned}
& z_{h}^{s}= \begin{cases}\left\lfloor\frac{\max _{h \in H} t_{h}^{s}-t_{h}^{s}}{\max _{h \in H} t_{h}^{s}-\min _{h \in H} t_{h}^{s}}\right\rfloor, & \text { if } L_{h}^{s}=1 \\
0, & \text { if } L_{h}^{s}=0,\end{cases} \\
& s \in S_{1}
\end{aligned}
$$

Moreover, constraints (17) and (18) show that the daily demand of available hospitals should be satisfied and the emergency demand should be probabilistically satisfied in case of disasters, respectively. $\alpha$ in the chance constraints (18) is the ratio of demand satisfaction, and constraints (18) can be transformed into corresponding deterministic linear constraints (23) and (24) [40]:

$$
\begin{aligned}
& \sum_{i \in I}\left[\left(y_{i h a b}^{s}+\sum_{b^{\prime} \in B L, b^{\prime} \neq b} q_{b b^{\prime}} \cdot y_{i h a b b^{\prime}}^{s}-x_{i} \cdot L_{h}^{s}\right.\right. \\
& \left.\left.\cdot\left(T+t_{i h}\right) \cdot d_{h a b}\right)\right]+s_{h a b} \geq z_{h}^{s} \cdot k_{a b \alpha}^{s}, \\
& h \in H ; a \in A ; b \in B L ; s \in S_{1}
\end{aligned}
$$

where

$$
\begin{aligned}
& k_{a b \alpha}^{s}=\sup \left\{k_{a b}^{s} \mid k_{a b}^{s}=\left(F_{a b}^{s}\right)^{-1}(1-\alpha)\right\}, \\
& \qquad s \in S_{1} ; a \in A ; b \in B L
\end{aligned}
$$

in which $F_{a b}^{s}$ is the distribution function of $\Delta T \cdot d_{a b}^{s}$, and $f_{a b}^{s}$ is the corresponding probability density function. A primary challenge in above modelling is to estimate $F_{a b}^{s}$ and $f_{a b}^{s}$, which will be discussed in next section in detail.

Finally, constraints (19) guarantee that the distribution flows through unselected blood bank candidate are zero. Constraints (20) show that the distribution flows to unavailable hospitals in disaster periods are zero. Constraints (21) specify the domains of the decision variables.

3.3. Bayesian Network (BN) and BN-Based Scenario Set. In this section, based on earthquakes, we propose the corresponding BN model to generate the scenario set $S$ used in our two-stage SP model. The variables used in the BN model can be referred to Table 6 . Obviously, variables $C, F$, and $L$ vary with different earthquakes. Variables $G$ and $D$ would influence the final demand directly. Hence, it is natural to consider these factors in our BN model. 


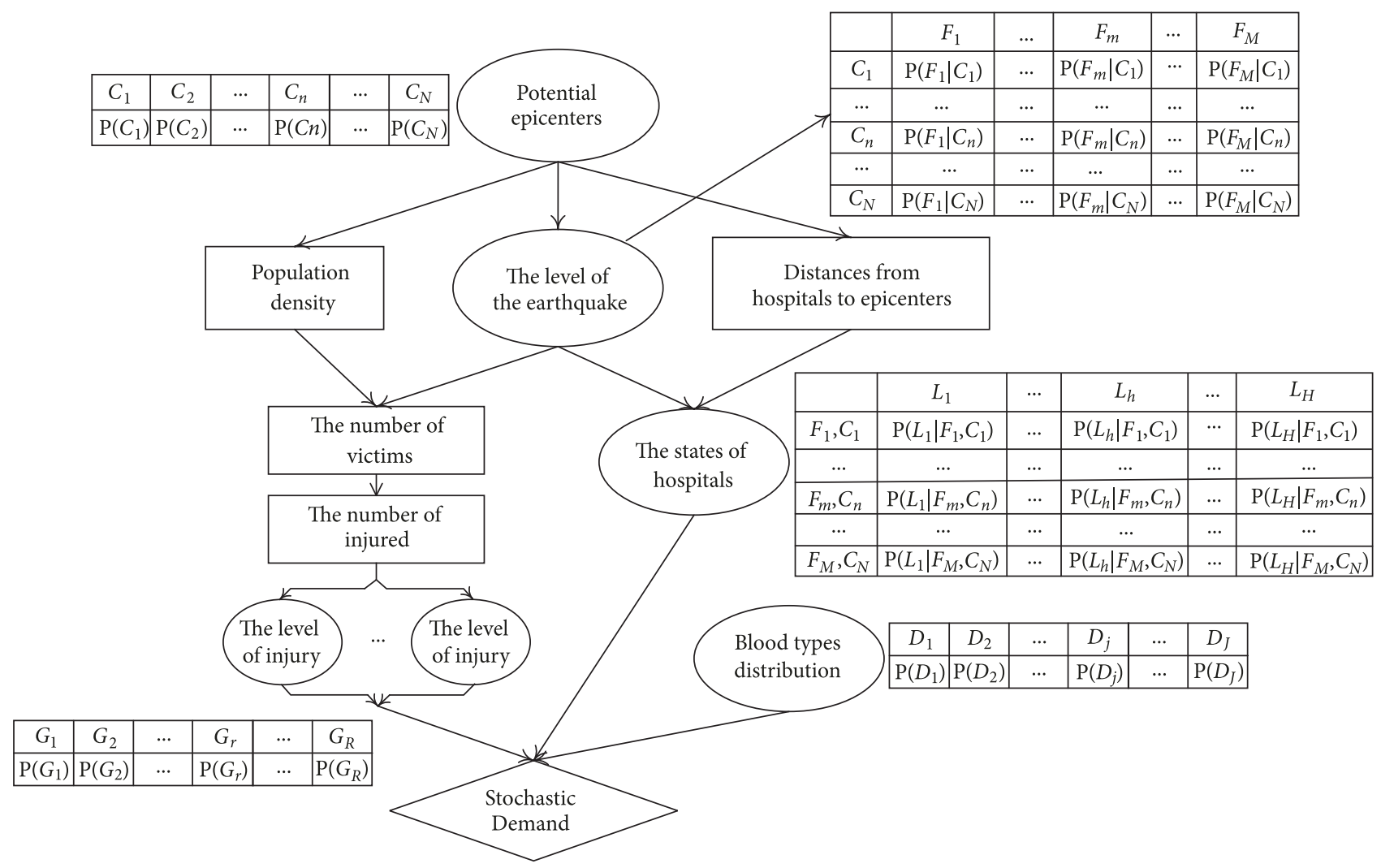

FIGURE 3: Proposed BN for the earthquake scenarios.

Thus, the proposed BN, as shown in Figure 3, consists of above five random variables (see the elliptical frame) contributing to stochastic blood demand (see the rhombic frame) by different intermediate variables (see the rectangular frame) for each blood product. The tables associated with these random variables in Figure 3 also show their possible states and corresponding possibilities.

Next, their inter-relationship can be quantified as follows.

(1) The States of Hospitals. That the hospitals may be unavailable in a disaster would obviously influence the effects of disaster relief. However, it is rarely considered in the BLNs related studies. Here, we assume the probability of the unavailability of a hospital is inversely proportional to its distance to the epicenter, being influenced by the level of the earthquake as well. This assumption is reasonable since the closer a hospital is to the epicenter, the higher the earthquake's level is, and the higher its unavailable probability is. Thus, the range normalization method is used to transfer the distance into the closed interval $[0,1]$, taking the level of the earthquake into account. Hence, the probability of the unavailability of a hospital is the conditional probability of $C$ and $F$ :

$$
p\left(L_{h}=0 \mid F_{m}, C_{n}\right)=\max \{0,
$$

$$
\begin{aligned}
& \left.\frac{d i s_{n h}-\max _{0 \leq \varepsilon \leq \vartheta_{F_{m}}}\left(\vartheta_{F_{m}}-\varepsilon\right)}{\min _{0 \leq \varepsilon \leq \vartheta_{F_{m}}}\left(\vartheta_{F_{m}}-\varepsilon\right)-\max _{0 \leq \varepsilon \leq \vartheta_{F_{m}}}\left(\vartheta_{F_{m}}-\varepsilon\right)}\right\} \\
& =\max \left\{0, \frac{d i s_{n h}-\vartheta_{F_{m}}}{0-\vartheta_{F_{m}}}\right\}, \\
& h \in H ; F_{m} \in F ; C_{n} \in C ; n=1, \ldots, N
\end{aligned}
$$

in which $C_{n}$ and $F_{m}$ are used to represent the events of set $C$ and $F$, respectively. $\vartheta_{F_{m}}$ is the maximum affected epicentral distance calculated according to the earthquake level $F_{m}$ and the given intensity, which results in facility or road damage [41]. Hence, we define the affected area as the circle with center point $C_{n}$ and a radius of $\vartheta_{F_{m}} . \varepsilon$ is the distance between epicenter and any point in the affected area. It is obvious that when the distance between epicenter and hospital is larger than $\vartheta_{F_{m}}$, the unavailable probability is zero. We use formula (26) to calculate the maximum epicentral distance $\left(\vartheta_{F_{m}}\right)$ when the level of the earthquake is $F_{m}$ with the given intensity $\left(\eta_{0}\right)$ [42].

$$
\begin{aligned}
\eta_{0}= & 0.514+1.5 F_{m}-0.00659 \vartheta_{F_{m}} \\
& -2.014 \lg \left(\vartheta_{F_{m}}+10\right), \quad F_{m} \in F
\end{aligned}
$$


(2) The Stochastic Blood Demand in the Earthquake. Notice that different potential epicenters may have distinctive possible levels of the earthquake. Thus, use $\mathrm{P}\left(F_{m} \mid C_{n}\right)$ to indicate the occurrence probability of level $F_{m}$ in epicenter $C_{n}$, which is known before disasters. For a given $F_{m}$, Lee [43] presents the following formula to calculate the corresponding intensity, denoted as Intensity ${ }_{m}$.

$$
\text { Intensity }_{m}=\frac{F_{m}-1.5}{0.58}, \quad F_{m} \in F
$$

Moreover, based on historical data in China, Ma [44] gives an empirical formula to evaluate the total number of affected victims (represented as $N V$ ), which includes the injured and the fatal, by corresponding the intensity as follows:

$$
\begin{aligned}
\ln N V_{n m}= & -11.346+0.855 \ln F_{m} \cdot \text { Density }_{n} \\
& +6.078 \ln \text { Intensity }_{m}, \quad F_{m} \in F, C_{n} \in C
\end{aligned}
$$

in which Density ${ }_{n}$ means the population density of epicenter $C_{n}$.

Then, with $N V_{n m}$, we can build its relationship with final stochastic blood demand by the following two steps.

At first, Wyss and Trendafiloski [45] use $R$ to express the ratio of the injured to the fatal and calculate corresponding $R s$ in some main areas and countries from historical statistics. For example, the value of $R$ in China is 12.8 . Thus, the number of the injured $\Omega_{n m}$ can be obtained as follows.

$$
\Omega_{n m}=N V_{n m} \cdot \frac{R}{1+R}
$$

Second, for each injured, the demand for blood products $a$ per hour also could be stochastic, denoted as $x_{1 a}$ with the probability $\mathrm{P}\left(G_{1}\right)$ or $x_{2 a}$ with the probability $P\left(G_{2}\right)$ which can be obtained by historical data. By Central Limit Theorem, the total demand for blood product $a$ is approximately normally distributed especially when $\Omega_{n m}$ is big enough. Let $X_{a}$ be the demand for blood product $a$ of each injured person per hour, and the mean and variance of total blood demand are explained as formulas (30) and (31):

$$
\begin{aligned}
E\left(\Omega_{n m} X_{a}\right) & =\Omega_{n m} \cdot E\left(X_{a}\right) \\
& =\Omega_{n m} \cdot\left[P\left(G_{1}\right) x_{1 a}+P\left(G_{2}\right) x_{2 a}\right] \\
D\left(\Omega_{n m} X_{a}\right) & =\Omega_{n m} \cdot D\left(X_{a}\right) \\
& =\Omega_{n m} \cdot P\left(G_{1}\right) \cdot P\left(G_{2}\right) \cdot\left(x_{1 a}-x_{2 a}\right)^{2}
\end{aligned}
$$

The event that an earthquake occurs at potential epicenter $C_{n}$ at level $F_{m}$ constitutes a part of scenario $s$. Therefore, the demand for blood product $a$ per hour under scenario $s$ (denoted as $d_{a}^{s}(\mathrm{U} / \mathrm{h})$ ) can be represented as

$$
d_{a}^{s} \sim N\left(\Omega_{n m} E\left(X_{a}\right), \Omega_{n m} D\left(X_{a}\right)\right)
$$

According to Peng [46] and Chen [47], the blood type distribution may differ from region to region. Also, in China, rapid economic development results in obvious human migration, making the blood type distribution in a specific region fluctuate. Hence, for the people in a region, the blood type distribution is also stochastic. Thus, for each blood type distribution $D_{j} \in D$, denote $p_{b j}$ as the proportion of blood type $b$ in all the injured, and $\sum_{b \in B L} p_{b j}=1$. Assume $D_{j}$ occurs in scenario $s$; thus, the corresponding emergency demand per hour for blood product $a$ with type $b$, i.e., $d_{a b}^{s}$, can be expressed as

$$
d_{a b}^{s}=p_{b j} \cdot d_{a}^{s}
$$

As we mentioned before, the blood prepositioned in the BLN needs to satisfy the blood demand of the transfusion time $\Delta T$. Thus, the emergency demand for blood product $a$ with type $b$ is $\Delta T d_{a b}^{s}$, and its probability density function $f_{a b}^{s}$ used in formula (24) can be expressed as

$$
\begin{array}{r}
f_{a b}^{s}=\frac{1}{\sqrt{2 \pi} \sigma} \exp \left(-\frac{\left(d_{a b}^{s}-\Delta T \cdot p_{b j} \cdot \Omega_{n m} E\left(X_{a}\right)\right)}{2 \Delta T^{2} \cdot p_{b j}^{2} \cdot \Omega_{n m} D\left(X_{a}\right)}\right), \\
s \in S_{1} ; a \in A ; b \in B L
\end{array}
$$

When the hospital $h$ is unavailable (i.e., $L_{h}=0$ ), the blood bank cannot replenish it at the present period. Hence, the states of hospitals will not change the emergency demand but would influence the final total demand.

Finally, one disaster scenario $s$ composed by $\left\{C_{n}, F_{m}\right.$, $\left.G_{r}, D_{j}, L_{h}\right\}$ and its impacts can be generated from the above procedure. Its corresponding probability is determined by the product of the probability of each random variable as

$$
\begin{aligned}
p^{s}= & P\left(C_{n}\right) \times P\left(F_{m} \mid C_{n}\right) \times P\left(G_{r}\right) \times P\left(D_{j}\right) \\
& \times \prod_{h=1}^{H} P\left(L_{h} \mid F_{m}, C_{n}\right), \quad s \in S_{1}
\end{aligned}
$$

All disaster scenarios constitute disaster scenario set $S_{1}$. Although there are multiple hospitals and potential epicenters, we assume that at least one hospital is available and at most one point causes earthquake. Therefore, $\left|S_{1}\right|=|C| \times$ $|F| \times|G| \times|D| \times\left(2^{|H|}-1\right)$, in which the symbol $|\bullet|$ is used to indicate the number of elements in set "•". Notice, there is another scenario where no earthquake happens. Hence, the dimensionality of the whole scenario set $S$ is $|S|=\left|S_{1}\right|+1$.

\section{Simulation}

In this section, we implement simulation study based on earthquakes in Sichuan Province, China. In order to evaluate the impacts of the $\mathrm{BN}$ model, we would test and compare simulation cases under different scenario sets and parameters, such as the probability of disasters and the unit inventory holding cost.

\subsection{The Construction of Disaster Scenario Sets Based on BN}

4.1.1. The Simulation Background. We would introduce our simulation background based on the Longmenshan earthquake zone in Sichuan, China. 


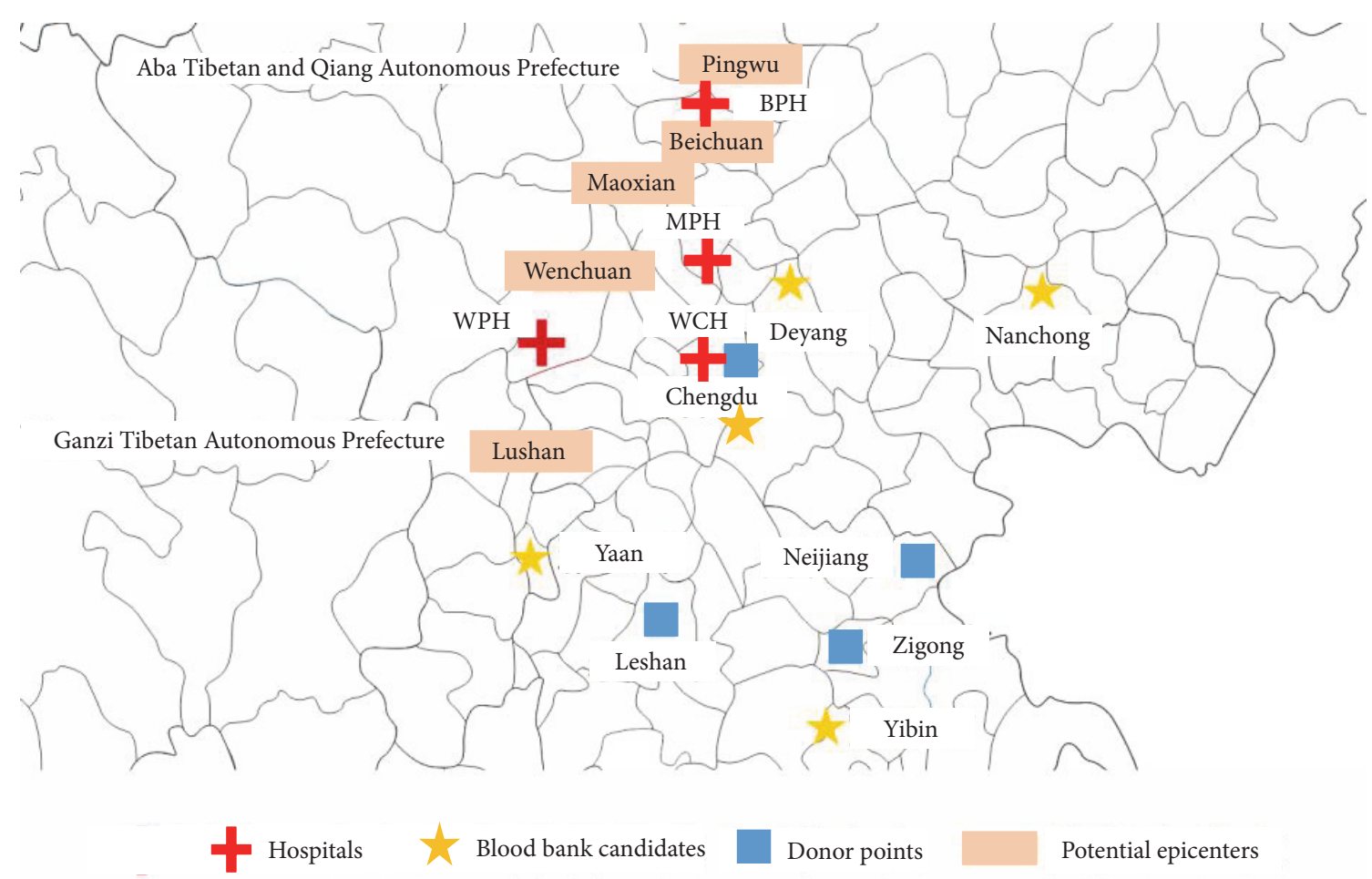

FIGURE 4: The geographic picture of blood bank supply chain.

TABLE 7: The parameters of the blood bank candidate $i$.

\begin{tabular}{lcc}
\hline $\begin{array}{l}\text { Blood bank } \\
\text { candidates } i\end{array}$ & $\begin{array}{c}\text { Fixed cost } \\
c_{i}\left(10^{7} \mathrm{RMB}\right)\end{array}$ & $\begin{array}{c}\text { Inventory holding } \\
\text { cost } r_{i}(\mathrm{RMB} / \mathrm{U} \cdot \mathrm{h})\end{array}$ \\
\hline Chengdu & 1.2 & 0.012 \\
Yibin & 1.1 & 0.01 \\
Nanchong & 1.08 & 0.008 \\
Yaan & 1.05 & 0.006 \\
Deyang & 1.12 & 0.007 \\
\hline
\end{tabular}

In the operational stage, each period $T$ is set as a week. The planning horizon is 10 years (i.e., $\Phi \approx 520$ weeks). The transfusion time $\Delta T$ is set to be $2(\mathrm{~h})$, because the transfusion duration of each injured at one operation is about 2 hours according to clinical data. The ratio of demand satisfaction $\alpha$ of blood supply is $95 \%$.

The random epicenters, donor points, blood bank candidates, and hospitals are shown in the Figure 4.

Firstly, their parameters are elaborated below.

(1) Potential epicenters set $C=$ (Wenchuan, Beichuan, Maoxian, Lushan, Pingwu) are all in the Longmenshan earthquake zone. The corresponding population densities are $0.0027,0.0071,0.0028,0.0088$, and 0.0031 (million $/ \mathrm{km}^{2}$ ), respectively (see http://data.stats.gov.cn/index.htm).

(2) Let the blood bank candidates set $I$ as (Chengdu, Yibin, Nanchong, Yaan, Deyang), and each candidate's corresponding fixed cost and inventory holding cost are displayed in Table 7.
(3) Considering that the number of blood donors is positively correlated with the population density, top four cities in population density in Sichuan Province are designated as main blood donation points. Thus, by World Population Network [48], we have $K=$ (Chengdu, Neijiang, Zigong, Leshan).

(4) Set $H$ is composed by hospitals which have superior medical conditions or are close to the random epicenters. Thus $H=$ (West China Hospital, Beichuan People's Hospital, Wenchuan People's Hospital, Mianzhu People's Hospital) and is denoted as $H=(\mathrm{WCH}, \mathrm{BPH}, \mathrm{WPH}, \mathrm{MPH})$ for short. The unit inventory holding cost $\left(u_{h}\right)$ of the hospitals are $(0.02$, $0.015,0.008,0.01) \mathrm{RMB} / \mathrm{U} \cdot \mathrm{h}$, respectively.

Secondly, the parameters of blood products are given as follows.

(1) The multiproduct set is denoted as $A=$ (Plasma, Red blood cells, Platelets), and the multitype set is denoted as $B L=$ $(\mathrm{A}, \mathrm{B}, \mathrm{AB}, \mathrm{O})$. Letters $\mathrm{A}, \mathrm{B}, \mathrm{AB}$, and $\mathrm{O}$ are used to represent $\mathrm{A}+, \mathrm{B}+, \mathrm{AB}+$, and $\mathrm{O}+$ blood types and the negative cases are ignored because people with blood types $\mathrm{A}_{-}, \mathrm{B}-, \mathrm{AB}-$, and $\mathrm{O}-$ are rare. The mutual substitution relationship of different blood types can be referred to Table 5 .

(2) By "The Minister of Health of the People's Republic of China [49]", the lifespans $l_{a}(a \in \mathrm{A})$ of plasma (thawed), red blood cells, platelets during transportation are set to be 1,21 , and 1 day, respectively.

(3) Table 8 gives the maximum quantity of blood that each donor point can supply per period $T$ [50].

(4) By Wang et al. [51], the daily deterministic demand of each hospital is given in Table 9. 
TABLE 8: The maximum supply quantity $\left(f_{k a b}\right)(\mathrm{U})$ of donor point $k$ per period $T$.

\begin{tabular}{lcccccccccccc}
\hline \multirow{2}{*}{ Donor point $k$} & \multicolumn{4}{c}{ Plasma } & \multicolumn{4}{c}{ Red blood cells } & \multicolumn{4}{c}{ Platelets } \\
& $\mathrm{A}$ & $\mathrm{B}$ & $\mathrm{AB}$ & $\mathrm{O}$ & $\mathrm{A}$ & $\mathrm{B}$ & $\mathrm{AB}$ & $\mathrm{O}$ & $\mathrm{A}$ & $\mathrm{B}$ & $\mathrm{AB}$ & $\mathrm{O}$ \\
\hline Chengdu & 435 & 377 & 103 & 569 & 274 & 238 & 65 & 359 & 122 & 106 & 29 & 160 \\
Neijiang & 279 & 242 & 66 & 366 & 176 & 153 & 42 & 231 & 79 & 68 & 19 & 103 \\
Zigong & 305 & 264 & 72 & 399 & 192 & 167 & 46 & 252 & 86 & 74 & 21 & 112 \\
Leshan & 112 & 97 & 27 & 146 & 71 & 61 & 17 & 92 & 32 & 28 & 8 \\
\hline
\end{tabular}

TABLE 9: The daily blood demand $\left(d_{h a b}\right)(\mathrm{U} / \mathrm{h})$ in hospital $h$.

\begin{tabular}{lccccc}
\hline \multirow{2}{*}{ Blood product $a$} & \multirow{2}{*}{ Blood type $b$} & \multicolumn{4}{c}{ Hospitals $h$} \\
& & WCH & BPH & WPH & MPH \\
\hline \multirow{3}{*}{ Plasma } & A & 3.050 & 0.213 & 0.081 & 1.191 \\
& $\mathrm{~B}$ & 2.644 & 0.184 & 0.070 & 1.032 \\
& $\mathrm{AB}$ & 0.718 & 0.050 & 0.019 & 0.280 \\
& $\mathrm{O}$ & 3.007 & 0.281 & 0.107 & 1.562 \\
\hline \multirow{3}{*}{ Red blood cells } & $\mathrm{A}$ & 1.932 & 0.137 & 0.052 & 0.755 \\
& $\mathrm{~B}$ & 1.675 & 0.118 & 0.045 & 0.655 \\
& $\mathrm{AB}$ & 0.455 & 0.032 & 0.012 & 0.178 \\
& $\mathrm{O}$ & 2.532 & 0.179 & 0.068 & 0.989 \\
\hline \multirow{3}{*}{ Platelets } & $\mathrm{A}$ & 0.829 & 0.058 & 0.022 & 0.324 \\
& $\mathrm{~B}$ & 0.719 & 0.050 & 0.019 & 0.281 \\
& $\mathrm{AB}$ & 0.195 & 0.013 & 0.005 & 0.076 \\
& $\mathrm{O}$ & 1.087 & 0.076 & 0.029 & 0.425 \\
\hline
\end{tabular}

TABLE 10: The transportation time $\left(t_{k i}\right)(\mathrm{h})$ from the donor point $k$ to the blood bank candidates $i$.

\begin{tabular}{lcccc}
\hline \multirow{2}{*}{ Blood bank candidate $i$} & \multicolumn{4}{c}{ Donor point $k$} \\
& Chengdu & Neijiang & Zigong & Leshan \\
\hline Chengdu & 0.15 & 2.5 & 3 & 2 \\
Yibin & 3.2 & 1.5 & 1 & 2 \\
Nanchong & 3 & 2.2 & 2.7 & 3.5 \\
Yaan & 1.8 & 2.8 & 2.8 & 1.5 \\
Deyang & 1.5 & 2.7 & 3 & 2.8 \\
\hline
\end{tabular}

Furthermore, the parameters of transportation are given as follows:

(1) The transportation velocity $v$ is assumed as an average value of $60(\mathrm{~km} / \mathrm{h})$ in this paper, and the transportation fee $e$ is set as $0.07(\mathrm{RMB} / \mathrm{km} \cdot \mathrm{U})$ according to Jabbarzadeh et al. [22].

(2) The transportation time from donor points to blood bank candidates and from blood bank candidates to hospitals and the distance from potential epicenters to hospitals, which can be obtained from Google Maps, are given in Tables 10-12, respectively.

4.1.2. The Scenario Sets Based on BN. Based on above parameters, we show how to construct scenario sets by the proposed $\mathrm{BN}$ model. Notice the final scenario set may vary due to the states and possibilities of the random factors. Hence, we only give one scenario set here. Other kinds of scenario sets can be given in similar way.
TABLE 11: The transportation time $\left(t_{i h}\right)(\mathrm{h})$ from the blood bank candidate $i$ to the hospital $h$.

\begin{tabular}{lccccc}
\hline \multirow{2}{*}{ Hospitals $h$} & \multicolumn{5}{c}{ Blood bank candidate $i$} \\
& Chengdu & Yibin & Nanchong & Yaan & Deyang \\
\hline WCH & 0.15 & 3.2 & 2.8 & 2 & 1.5 \\
BPH & 3 & 5.2 & 4.1 & 3.7 & 1.7 \\
WPH & 2.3 & 4.5 & 3.8 & 3 & 2.5 \\
MPH & 1.8 & 4 & 2.4 & 2.5 & 0.9 \\
\hline
\end{tabular}

TABLE 12: The distance $\left(d i s_{n h}\right)(\mathrm{km})$ from the epicenter $n$ to the hospital $h$.

\begin{tabular}{lcccc}
\hline \multirow{2}{*}{ Epicenter $n$} & \multicolumn{4}{c}{ Hospital $h$} \\
& WCH & BPH & WPH & MPH \\
\hline Wenchuan & 147 & 130 & 5 & 173 \\
Beichuan & 140 & 5 & 232 & 53.4 \\
Maoxian & 187 & 89.1 & 41.9 & 212 \\
Lushan & 170 & 305 & 261 & 241 \\
Pingwu & 273 & 124 & 368 & 211 \\
\hline
\end{tabular}

(1) For $C=$ (Wenchuan, Beichuan, Maoxian, Pingwu, Lushan), we give the probabilities of the earthquake as $0.03,0.025,0.02,0.01$, and 0.015 , respectively. Thus, Table 13 could be obtained in which the corresponding probability of earthquakes in each single epicenter is shown. For example, the probability of a singlepoint earthquake in Wenchuan can be calculated as $\mathrm{P}\left(C_{1}\right) \times\left(1-\mathrm{P}\left(C_{2}\right)\right) \times\left(1-\mathrm{P}\left(C_{3}\right)\right) \times\left(1-\mathrm{P}\left(C_{4}\right)\right) \times\left(1-\mathrm{P}\left(C_{5}\right)\right)=$ 0.027953 .

Next, since at most one epicenter at each period is assumed, the normalized results are displayed in Table 14 .

(2) Let $F=(8,7,6.5,6)$ represent four levels, which are displayed in Table 15, and can be applied in calculating the intensity (refer to formulas (27)). Considering all potential epicenters are in the same earthquake zone, $\mathrm{P}\left(F_{m} \mid C_{i}\right)$ can be simplified to $\mathrm{P}\left(F_{m}\right)$. The corresponding values for each $F_{m}$ is $0.264,0.189,0.151$, and 0.396 , respectively [52].

(3) For each blood product, $p_{\mathrm{A}}, p_{\mathrm{B}}, p_{\mathrm{AB}}, p_{\mathrm{O}}$ are used to represent the proportion of blood types $A, B, A B$, and $\mathrm{O}$, respectively. Here, the blood type distribution, i.e., $p_{\mathrm{A}}: p_{\mathrm{B}}: p_{\mathrm{AB}}: p_{\mathrm{O}}$, of injured people is considered to be random and has two kinds of blood type distribution, i.e., $D=\left(D_{1}, D_{2}\right)$. Specifically, according to the mean blood type distribution in Sichuan Province [46], two equal-weighted distributions $D_{1}=0.33: 0.24: 0.07: 0.36$ and $D_{2}=0.32: 0.26: 0.08: 0.34$ are considered. 
TABLE 13: The probability of single epicenter and no disaster.

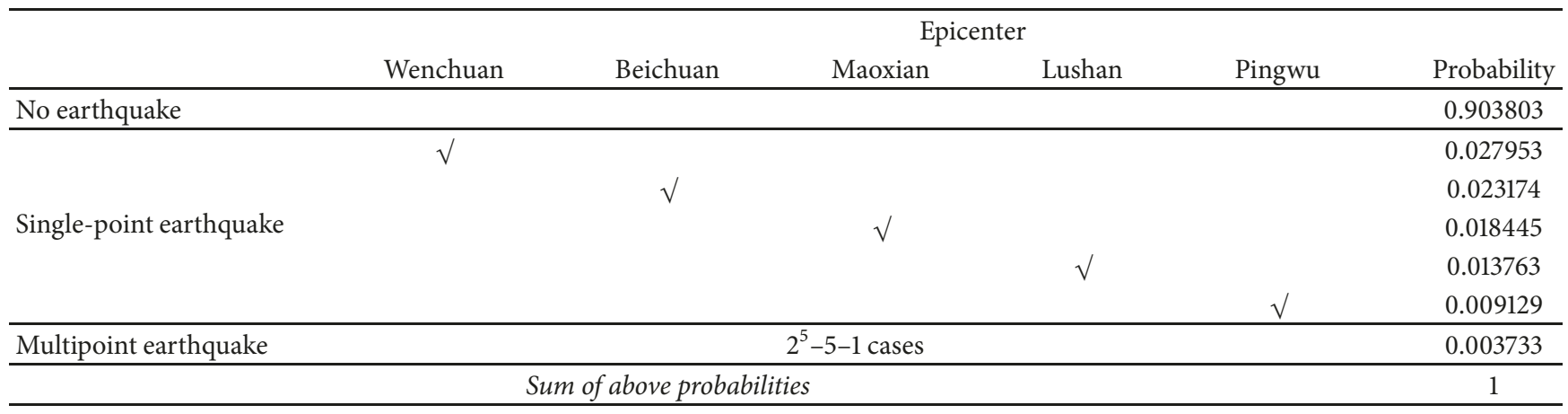

TABLE 14: The normalization of probability.

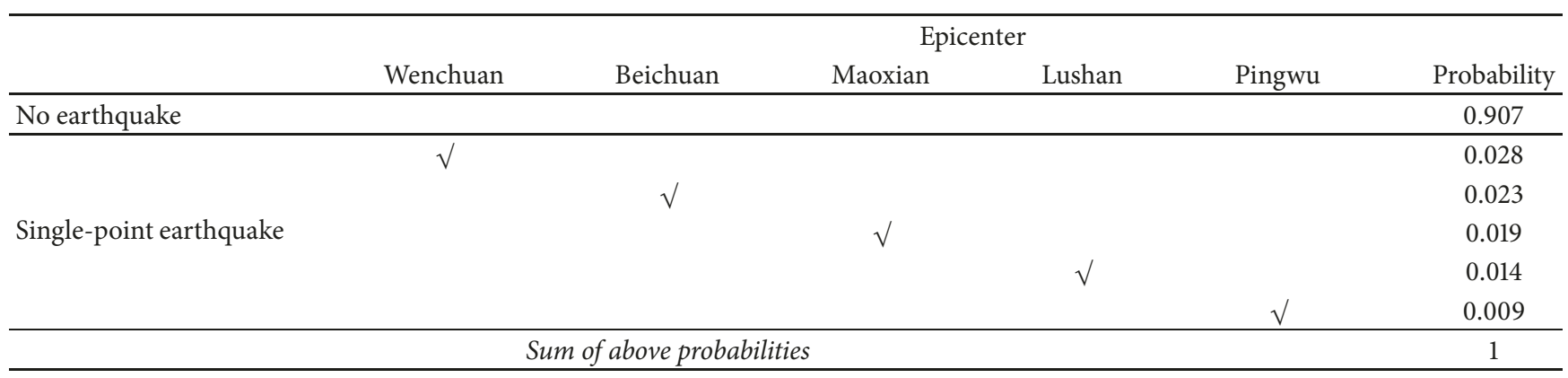

TABLE 15: The levels of the earthquake and set $F$.

\begin{tabular}{lccc}
\hline Rank & Magnitude & The level of the earthquake & Set $F$ \\
\hline I & $\geq 7.0$ & Especially large earthquake & $F_{1}=8$ \\
II & $6.5 \sim 7.0$ & Large earthquake & $F_{2}=7$ \\
III & $6.0 \sim 6.5$ & Relatively large earthquake & $F_{3}=6.5$ \\
IV & $5.0 \sim 6.0$ & General earthquake & $F_{4}=6$ \\
\hline
\end{tabular}

TABLE 16: The quantity of two-level demand for each blood product (per injured).

\begin{tabular}{lcc}
\hline Blood product & Rank & Quantity(U/h) \\
\hline \multirow{2}{*}{ Plasma } & High & 0.225 \\
& Low & 0.15 \\
\multirow{2}{*}{ Red blood cells } & High & 1.136 \\
& Low & 0.522 \\
\multirow{2}{*}{ Platelets } & High & 0.182 \\
& Low & 0.094 \\
\hline
\end{tabular}

(4) Two demand levels, seriously and slightly, are assumed because the blood demand varies from person to person by injury level difference. Two random proportions, $G=\left(G_{1}, G_{2}\right)$, of two demand levels are considered, which are $G_{1}=0.3: 0.7$ and $G_{2}=0.4: 0.6$. The probabilities of two random proportions (i.e., $\left.\mathrm{P}\left(G_{r}\right), r=1,2\right)$ are both 0.5 . Table 16 displays the corresponding quantities of blood demands $[53,54]$ for each level $\left(x_{1 a}, x_{2 a}, a \in A\right)$.

(5) Utilize formulas (26) to calculate the maximum epicentral distance with given $\eta_{0}$ as VI [41]. Recall that at least one hospital is available in each scenario. Hence, the conditional unavailable probability of each hospital under the combination of set $C$ and $F$, which can be calculated by formulas (25), is shown in Table 17.

Till now, Figure 5 shows the scenario tree for each epicenter which is constructed according to parameters described above. The size of disaster scenarios set $S_{1}$ is theoretically up to $1200\left(=\left|S_{1}\right|=|C| \times|F| \times|G| \times|D| \times\left(2^{|H|}-1\right)=5 \times 4 \times 2\right.$ $\left.\times 2 \times\left(2^{4}-1\right)=5 \times 240\right)$, where the probability $p_{s}\left(s \in S_{1}\right)$ can be given by formulas (35). We denoted this dataset as 1200_1.

Moreover, notice $S_{1}$ varies with the random factors. In order to test the impacts of different $S_{1}$ on the decisions, we consider more possible values of the proportions of two demand levels $(G)$ and the blood type distributions $(D)$. Specifically, the proportions $G_{3}=0.5: 0.5, G_{4}=0.6: 0.4$, $G_{5}=0.7: 0.3$ and the distribution $D_{3}=0.318: 0.25: 0.082: 0.35$ are considered. Thus, different scenario sets can be obtained as in Table 18. In the case of 1200 or 3600 scenarios, we only consider the extreme situations where emergency demand is extremely low $\left(G_{1}, G_{2}\right)$ or $\left(G_{1}, G_{2}, G_{3}, G_{4}\right)$ and high $\left(G_{3}\right.$, $\left.G_{4}\right)$ or $\left(G_{2}, G_{3}, G_{4}, G_{5}\right)$, respectively. Three combinations of blood type distribution are also incorporated into datasets with 1200 scenarios, resulting totally in 6 datasets. The case dataset $\left|S_{1}\right|=4500$ is the combination of all possible values of random variables we mentioned above; hence, there is only one corresponding dataset.

\subsection{The Computational Results and Sensitivity Analysis}

4.2.1. Computational Results of All Scenario Sets. Assigning each dataset to the SP model which can be optimally solved by IBM ILOG CPLEX 12.6.3, the corresponding recourse 
TABLE 17: The conditional unavailable probability of hospitals $\left(\mathrm{P}\left(L_{h}=0 \mid F_{m}, C_{n}\right)\right)$.

\begin{tabular}{|c|c|c|c|c|c|c|c|c|c|c|c|c|c|c|c|c|}
\hline \multirow{3}{*}{ Epicenter $($ Set $C)$} & \multicolumn{16}{|c|}{ The level of the earthquake (Set $F$ ) } \\
\hline & \multicolumn{4}{|c|}{ Rank IV (6) } & \multicolumn{4}{|c|}{ Rank III (6.5) } & \multicolumn{4}{|c|}{ Rank II (7) } & \multicolumn{4}{|c|}{ Rank I (8) } \\
\hline & $\mathrm{WCH}$ & $\mathrm{BPH}$ & $\mathrm{WPH}$ & $\mathrm{MPH}$ & $\mathrm{WCH}$ & $\mathrm{BPH}$ & WPH & $\mathrm{MPH}$ & $\mathrm{WCH}$ & $\mathrm{BPH}$ & WPH & $\mathrm{MPH}$ & $\mathrm{WCH}$ & $\mathrm{BPH}$ & WPH & $\mathrm{MPH}$ \\
\hline Wenchuan & 0 & 0 & 0.909 & 0 & 0 & 0 & 0.95 & 0 & 0.065 & 0.173 & 0.968 & 0 & 0.513 & 0.57 & 0.983 & 0.427 \\
\hline Beichuan & 0 & 0.909 & 0 & 0.029 & 0 & 0.95 & 0 & 0.464 & 0.11 & 0.968 & 0 & 0.66 & 0.537 & 0.983 & 0.232 & 0.823 \\
\hline Maoxian & 0 & 0 & 0.238 & 0 & 0 & 0.105 & 0.579 & 0 & 0 & 0.433 & 0.734 & 0 & 0.381 & 0.705 & 0.861 & 0.298 \\
\hline Lushan & 0 & 0 & 0 & 0 & 0 & 0 & 0 & 0 & 0 & 0 & 0 & 0 & 0.437 & 0 & 0.136 & 0.202 \\
\hline Pingwu & 0 & 0 & 0 & 0 & 0 & 0 & 0 & 0 & 0 & 0.211 & 0.978 & 0 & 0.096 & 0.589 & 0 & 0.301 \\
\hline
\end{tabular}

TABLE 18: Characteristics of all datasets.

\begin{tabular}{|c|c|c|c|}
\hline \multirow{2}{*}{$\begin{array}{l}\text { The dimensionality } \\
\text { of the scenario sets }\end{array}$} & \multirow{2}{*}{ Dataset's name } & \multicolumn{2}{|c|}{ Random factors } \\
\hline & & $G$ & $D$ \\
\hline \multirow{6}{*}{$-S_{1}-=1200$} & 1200_1 & $\left(G_{1}, G_{2}\right)$ & $\left(D_{1}, D_{2}\right)$ \\
\hline & 1200_2 & $\left(G_{1}, G_{2}\right)$ & $\left(D_{1}, D_{3}\right)$ \\
\hline & $1200 \_3$ & $\left(G_{1}, G_{2}\right)$ & $\left(D_{2}, D_{3}\right)$ \\
\hline & $1200 \_4$ & $\left(G_{4}, G_{5}\right)$ & $\left(D_{1}, D_{2}\right)$ \\
\hline & $1200 \_5$ & $\left(G_{4}, G_{5}\right)$ & $\left(D_{1}, D_{3}\right)$ \\
\hline & $1200 \_6$ & $\left(G_{4}, G_{5}\right)$ & $\left(D_{2}, D_{3}\right)$ \\
\hline \multirow{2}{*}{$-S_{1}-=3600$} & $3600 \_1$ & $\left(G_{1}, G_{2}, G_{3}, G_{4}\right)$ & $\left(D_{1}, D_{2}, D_{3}\right)$ \\
\hline & $3600 \_2$ & $\left(G_{2}, G_{3}, G_{4}, G_{5}\right)$ & $\left(D_{1}, D_{2}, D_{3}\right)$ \\
\hline$-S_{1}-=4500$ & $4500 \_1$ & $\left(G_{1}, G_{2}, G_{3}, G_{4}, G_{5}\right)$ & $\left(D_{1}, D_{2}, D_{3}\right)$ \\
\hline
\end{tabular}

TABLE 19: The decisions of maximum inventory and emergency stock of the blood bank.

\begin{tabular}{|c|c|c|c|c|c|c|c|}
\hline \multicolumn{8}{|c|}{ The prespecified blood bank: Deyang } \\
\hline \multirow{12}{*}{$\begin{array}{c}\text { Maximum } \\
\text { inventory } \\
C_{i a b}(\mathrm{U})\end{array}$} & \multirow{4}{*}{ Plasma } & A & 4092.21 & \multirow{12}{*}{$\begin{array}{c}\text { Emergency } \\
\text { stock } \\
s s_{i a b}(\mathrm{U})\end{array}$} & \multirow{4}{*}{ Plasma } & A & 3379.45 \\
\hline & & $\mathrm{B}$ & 2460.53 & & & $\mathrm{~B}$ & 1821.26 \\
\hline & & $\mathrm{AB}$ & 766.14 & & & $\mathrm{AB}$ & 598.29 \\
\hline & & $\mathrm{O}$ & 6154.89 & & & $\mathrm{O}$ & 5220.76 \\
\hline & \multirow{4}{*}{ Red blood cells } & A & 4914.84 & & \multirow{4}{*}{ Red blood cells } & A & 4428.12 \\
\hline & & $\mathrm{B}$ & 3090.94 & & & $\mathrm{~B}$ & 2701.67 \\
\hline & & $\mathrm{AB}$ & 204.17 & & & $\mathrm{AB}$ & 98.43 \\
\hline & & $\mathrm{O}$ & 9402.44 & & & $\mathrm{O}$ & 8721.99 \\
\hline & \multirow{4}{*}{ Platelets } & A & 2842.62 & & \multirow{4}{*}{ Platelets } & A & 2633.79 \\
\hline & & B & 1212.25 & & & $\mathrm{~B}$ & 1029.25 \\
\hline & & $\mathrm{AB}$ & 596.31 & & & $\mathrm{AB}$ & 549.32 \\
\hline & & $\mathrm{O}$ & 4543.67 & & & $\mathrm{O}$ & 4269.8 \\
\hline
\end{tabular}

problem (RP) solution can be obtained. Taking the dataset 1200_1 as the example, in its RP solution, Deyang is selected as the blood bank. The planning and daily operational cost (first four lines in formula (4)) is $1.15 \times 10^{8}(\mathrm{RMB})$ and the disaster rescue cost (last line in formula (4)) is $5.32 \times 10^{6}$ (RMB), totaling $1.21 \times 10^{8}(\mathrm{RMB})$.

Specifically, Table 19 shows the maximum inventory and the emergency stock of the selected blood bank, i.e., Deyang, in this RP solution. Table 20 gives the distribution flow from Deyang to each hospital. The emergency stock in each hospital is shown in Table 21.
Notice there is no prepositioning emergency stock in WCH as shown in Table 21, since it is closer to the selected blood bank location Deyang than any potential epicenter. The blood bank can supply emergency blood timely; thus, no prepositioning inventory in $\mathrm{WCH}$ is required.

Similarly, other datasets can also be optimally calculated and Deyang is always selected as final blood bank, while the inventory results would be different. Specifically, the emergency stock in both blood bank and hospitals of each dataset is shown in Figure 6. It is obvious that more emergency stock will be incurred in both the blood bank and the hospitals 


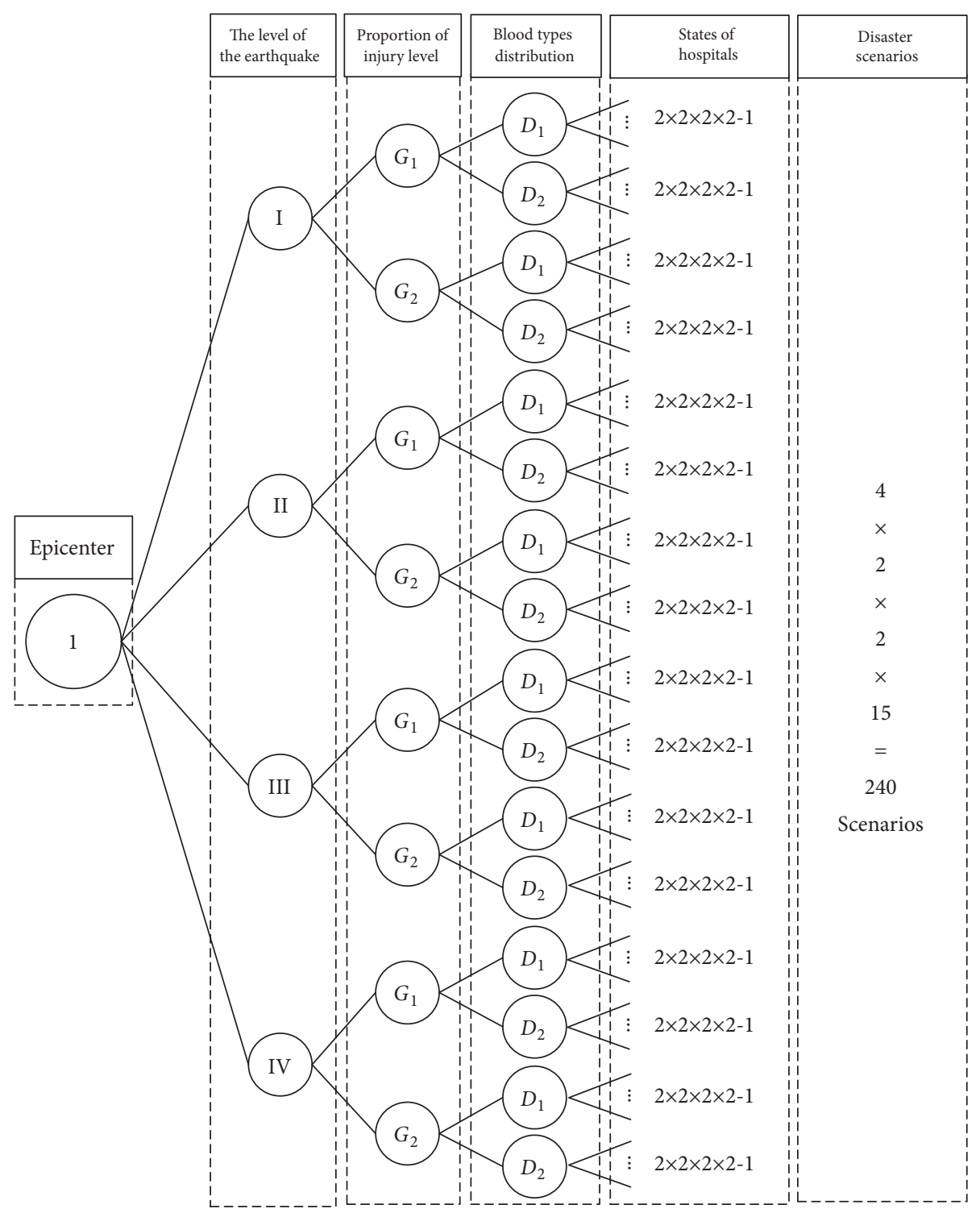

FIGURE 5: The scenario tree for one epicenter in the dataset 1200_1.

TABLE 20: The distribution flow $\left(w_{\text {ihab }}\right)(\mathrm{U})$ from the central blood bank to hospital $h$ in each daily period.

\begin{tabular}{|c|c|c|c|c|c|}
\hline \multirow{2}{*}{ Blood product $a$} & \multirow{2}{*}{ Blood type $b$} & \multicolumn{4}{|c|}{ Hospital $h$} \\
\hline & & $\mathrm{WCH}$ & $\mathrm{BPH}$ & WPH & $\mathrm{MPH}$ \\
\hline \multirow{4}{*}{ Plasma } & $\mathrm{A}$ & 461.65 & 36.14 & 13.81 & 201.16 \\
\hline & B & 448.15 & 31.22 & 12.11 & 147.79 \\
\hline & $\mathrm{AB}$ & 108.83 & 8.49 & 3.24 & 47.29 \\
\hline & $\mathrm{O}$ & 577.87 & 47.69 & 18.24 & 290.33 \\
\hline \multirow{4}{*}{ Red blood cells } & $\mathrm{A}$ & 327.47 & 23.24 & 8.87 & 127.52 \\
\hline & B & 242.03 & 20.02 & 7.67 & 119.55 \\
\hline & $\mathrm{AB}$ & 77.12 & 5.43 & 2.05 & 21.14 \\
\hline & $\mathrm{O}$ & 471.43 & 30.38 & 11.59 & 167.04 \\
\hline \multirow{4}{*}{ Platelets } & $\mathrm{A}$ & 140.51 & 9.84 & 3.75 & 54.72 \\
\hline & $\mathrm{B}$ & 121.87 & 9.57 & 4.09 & 47.46 \\
\hline & $\mathrm{AB}$ & 33.05 & 1.11 & 0.00 & 12.84 \\
\hline & $\mathrm{O}$ & 184.25 & 12.90 & 4.94 & 71.78 \\
\hline
\end{tabular}




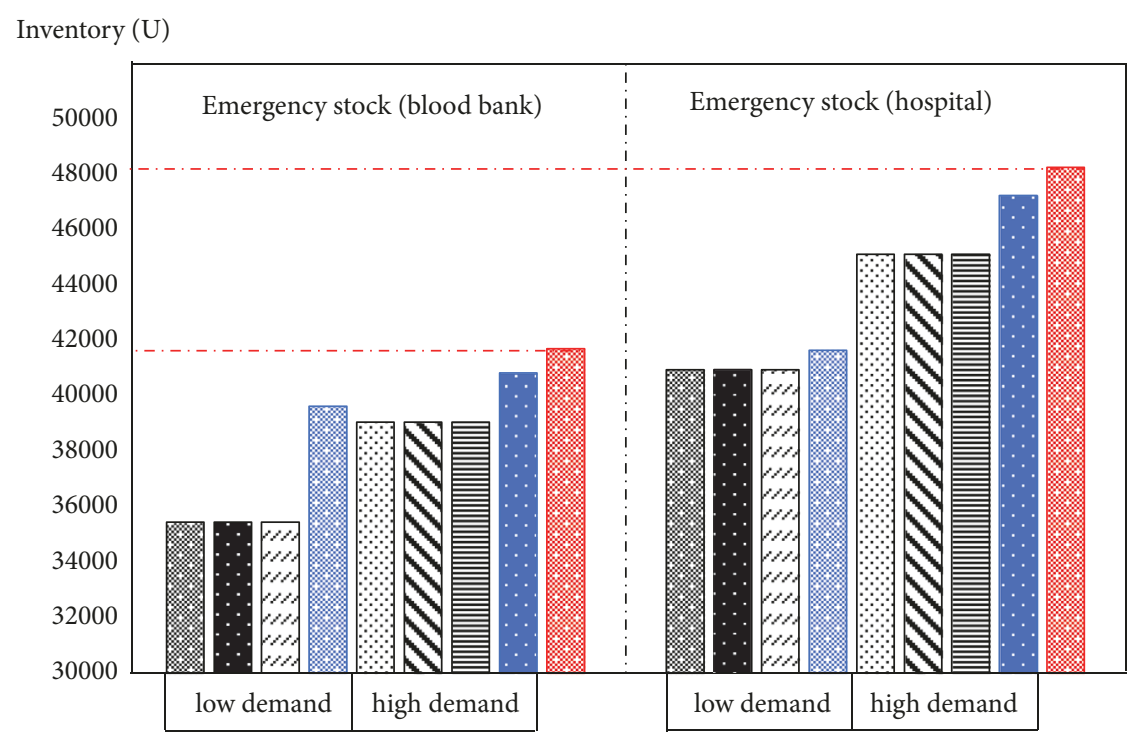

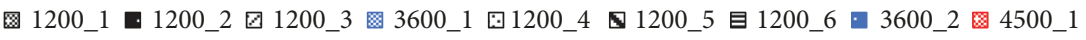

FIgURE 6: Total emergency stock in blood bank and all hospitals of different datasets.

TABLE 21: The emergency stock $\left(s_{\text {hab }}\right)(\mathrm{U})$ in hospital $h$.

\begin{tabular}{|c|c|c|c|c|c|}
\hline \multirow{2}{*}{ Blood product $a$} & \multirow{2}{*}{ Blood type $b$} & \multicolumn{4}{|c|}{ Hospital $h$} \\
\hline & & $\mathrm{WCH}$ & $\mathrm{BPH}$ & WPH & $\mathrm{MPH}$ \\
\hline \multirow{4}{*}{ Plasma } & $\mathrm{A}$ & 0 & 2451.57 & 1604.92 & 15.13 \\
\hline & B & 0 & 1932.33 & 1265.26 & 11.92 \\
\hline & $\mathrm{AB}$ & 0 & 596.29 & 391.02 & 3.68 \\
\hline & $\mathrm{O}$ & 0 & 2674.08 & 1750.46 & 16.5 \\
\hline \multirow{4}{*}{ Red blood cells } & A & 0 & 3547.9 & 2333.35 & 21.9 \\
\hline & B & 0 & 2799.65 & 1842.68 & 17.28 \\
\hline & $\mathrm{AB}$ & 0 & 870.96 & 576.4 & 5.37 \\
\hline & $\mathrm{O}$ & 0 & 870.96 & 2543.47 & 23.87 \\
\hline \multirow{4}{*}{ Platelets } & A & 0 & 1886.98 & 1236.37 & 11.64 \\
\hline & B & 0 & 1487.63 & 975.02 & 9.18 \\
\hline & $\mathrm{AB}$ & 0 & 459.76 & 302.01 & 2.83 \\
\hline & $\mathrm{O}$ & 0 & 2058.09 & 1348.35 & 12.70 \\
\hline
\end{tabular}

under the higher level of the injury, e.g., $G=\left(G_{4}, G_{5}\right)$. Besides, we can find that total emergency stock increases with the size of $S_{1}$. For example, the dataset with 4500 scenarios would result in the highest stock.

Moreover, for the datasets with the same $G$, different blood type distributions $(D)$ do not influence the extent of disasters, and, thus, the quantity of total inventory prepositioned in the BLN keeps unchanged. However, the inventory quantities with each blood type certainly would vary with different $D$. Due to space limitations, we do not list the results here.

In summary, the decision on blood bank location is consistent, but the different random variables would significantly influence the inventory decisions. Specifically, with the increase of the dimensionality of scenario set, more disaster scenarios, although their possibilities are getting smaller, would be taken into account. Thus, to handle these extreme situations, larger inventory would be prepositioned which makes the solution more conservative.

4.2.2. Sensitivity Analysis. This section further implements sensitivity analysis by changing (1) the disaster probability, i.e., $\mathrm{P}\left(C_{n}\right)$, which only influences the probability of each BNbased scenario rather than the dimensionality, and (2) the unit inventory holding (UIH) cost, a cost-related parameter which is irrelevant to the $\mathrm{BN}$.

(1) Sensitivity Analysis of the Disaster Probability. We focus on $\mathrm{P}\left(C_{n}\right)$ because it is usually not easy to estimate exactly. Considering earthquake is a small-probability event, we set the probabilities of the disaster happening in five potential epicenters to be $0.2,0.25,0.5,1,2,3,4,5$ times of the default value respectively. 
TABLE 22: Daily transportation cost and rescue cost under varying disaster probabilities (million).

\begin{tabular}{ccccccccr}
\hline The disaster probability change ratio & 0.2 & 0.25 & 0.5 & 1 & 2 & 3 & 4 \\
\hline & \multicolumn{7}{c}{ Daily transportation cost } \\
All datasets & 34.80 & 34.62 & 33.80 & 32.20 & 29.33 & 26.88 & 24.64 & 22.69 \\
\hline & \multicolumn{7}{c}{ Rescue cost } \\
1200_1 1200_3 & 1.14 & 1.44 & 2.74 & 5.32 & 9.96 & 13.87 & 17.38 & 20.51 \\
1200_4 1200_6 & 1.21 & 1.52 & 2.90 & 5.63 & 10.56 & 14.69 & 18.41 & 21.73 \\
$3600 \_1$ & 1.18 & 1.46 & 2.77 & 5.39 & 10.10 & 14.07 & 17.63 & 20.80 \\
$3600 \_2$ & 1.20 & 1.48 & 2.83 & 5.50 & 10.30 & 14.34 & 17.97 & 21.20 \\
$4500 \_1$ & 1.20 & 1.49 & 2.82 & 5.43 & 10.18 & 14.18 & 17.76 & 20.96 \\
\hline
\end{tabular}

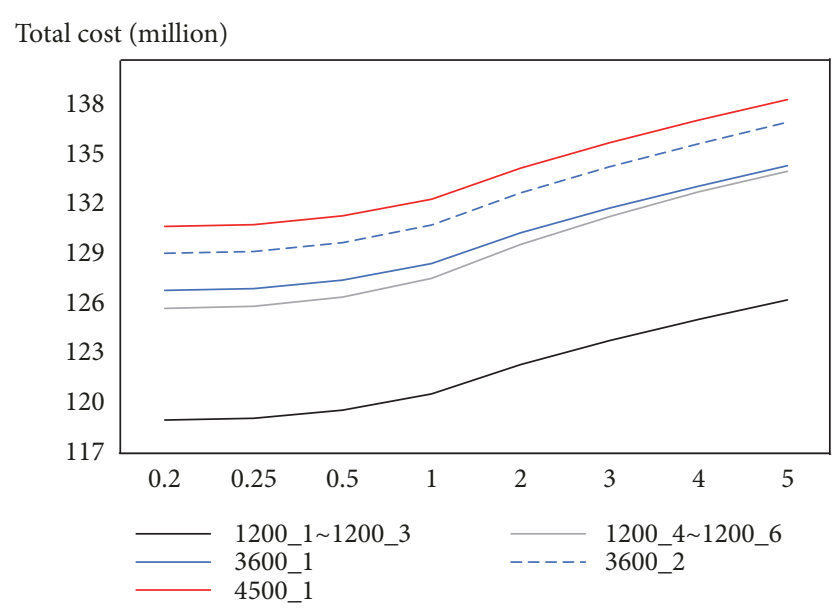

FIGURE 7: Total cost under varying disaster probabilities.

After calculation, it is found that, for each dataset, the decisions of blood bank location and emergency stock remain unchanged under the different disaster probabilities. And these two decisions are in the first stage, i.e., the planning stage, of two-stage SP which are what the decision-maker mainly care about. The reason is that $\mathrm{P}\left(C_{n}\right)$ only represents the occurrence probability but would not influence the extent of the disaster. Thus, it means, with the utilization of BN, we do not need a very exact disaster probability for the purpose of the BLN planning.

Since the decisions on location as well as emergency stock under different $\mathrm{P}\left(C_{n}\right)$ are the same, the inventory cost in each facility remains unchanged. However, the daily transportation cost, the rescue cost, and then the total cost would change along with the disaster probability as shown in Table 22 and Figure 7.

At first, for each disaster probability, the daily transportation cost of all datasets is identical. It is because their daily demand and the probability of normal scenario $p^{s_{0}}$ are the same. Moreover, the growth of the disaster probability would increase the probability of total disaster scenarios $\sum_{s \in S_{1}} p^{s}$ and thus decrease $p^{s_{0}}\left(=1-\sum_{s \in S_{1}} p^{s}\right)$. Based on formula (4), the daily transportation cost decreases and the rescue cost become larger when the disaster probability increases. Then, both of them work together and result in a higher total cost (see Figure 7).
(2) Sensitivity Analysis of the Unit Inventory Holding (UIH) Cost. To explore how the BN-unrelated parameters, i.e., the UIH cost, influence final decisions, sensitivity analysis is further implemented by varying $r_{i}(i \in I)$ and $u_{h}(h \in H)$ simultaneously. Their values can be $0.1,0.2, \ldots, 1.8$ times of their default values.

Firstly, Figure 8 shows the final location decisions in which the blood bank locations of all datasets change from Chengdu to Deyang with the increase of the UIH cost. The reason is that when the inventory cost is very low, the daily transportation cost would dominate the location decision. Thus, Chengdu is selected due to its geographical advantage. On the contrary, with the increase of this parameter, the decision-maker would choose a new blood bank, i.e., Deyang; thus, the inventory could be allocated in facilities with relatively lower cost. In addition, recall that, compared with 1200_1 1200_6, datasets with 3600 and 4500 scenarios would incur more inventory. Hence, they are more sensitive to the change of the UIH cost and thus would choose Deyang when this parameter is smaller than that of 1200 scenarios.

Moreover, it is found that the emergency stock prepositioned in the whole BLN, which is decided by the BN, remains unchanged under the different UIH costs. But the inventory in each facility may vary due the different location results. It also means the planning capacity of the blood bank is influenced by the UIH cost.

In summary, different from to the BN-related parameters which only affect the inventory decisions, the UIH cost would influence the location decision. Thus, it is necessary to measure the UIH cost exactly and analyze the location decision's sensitivity with respect to such cost-related parameters.

\section{The Expected Value Model}

To prove the effectiveness of the proposed two-stage SP model, in this section, we give the corresponding so-called Expected Value (EV) Model, in which the random variable is replaced by the expected value, and compare the results of two models.

5.1. The Formulation of the Expected Value Model. At first, we reformulate the two-stage SP in Section 3.2 to give the EV version as follows. The objective function (36) is the sum of cost in the planning stage and the daily periods of the 


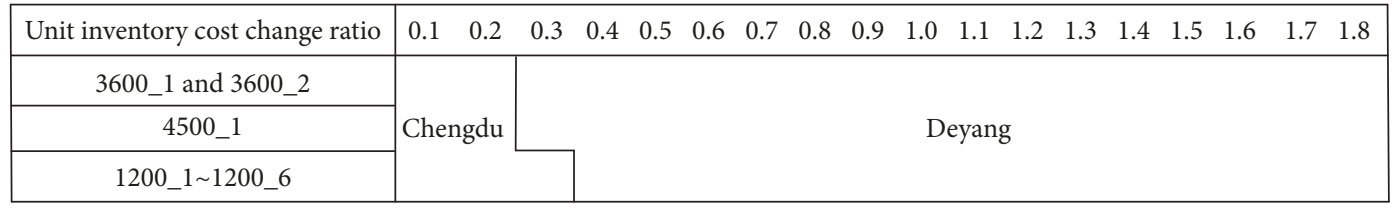

FIGURE 8: Blood bank location of each dataset under varying UIH costs.

operational stage, as well as the transportation cost of the expected value of blood demand when the disaster happens.

$$
\begin{aligned}
E V & =\min \sum_{i \in I} c_{i} \cdot x_{i}+\Phi \cdot\left\{T \cdot \sum _ { i \in I } \left[r_{i}\right.\right. \\
& \left.\cdot \sum_{k \in K} \sum_{a \in A} \sum_{b \in B L} \bar{g}_{k i a b}\right]+T \cdot \sum_{h \in H}\left[u_{h}\right. \\
& \left.\cdot \sum_{a \in A} \sum_{b \in B L}\left(\sum_{i \in I} \frac{x_{i} \cdot\left(T+t_{i h}\right) \cdot d_{h a b}}{2}+s_{h a b}\right)\right]+e \cdot v \\
& \left.\cdot \sum_{i \in I} \sum_{k \in K}\left(t_{k i} \cdot \sum_{a \in A} \sum_{b \in B L} \bar{g}_{k i a b}\right)\right\}+\Phi \cdot e \cdot v \\
& \cdot \sum_{i \in I} \sum_{h \in H}\left(t_{i h} \cdot \sum_{a \in A} \sum_{b \in B L}\left(w_{\text {ihab }}+z_{\text {ihab }}+\sum_{b^{\prime} \in B L, b^{\prime} \neq b}\left(w_{\text {ihabb' }}^{\prime}+z_{\text {ihabb }}^{\prime}\right)\right)\right)
\end{aligned}
$$

In the EV model, in addition to the original constraints (5) (7), (9), (12), and (13), new constraints (37) (41) are added as follows:

$$
\begin{aligned}
& \sum_{k \in K} \bar{g}_{k i a b} \leq C_{i a b}, \quad i \in I ; a \in A ; b \in B L \\
& 0 \leq \sum_{i \in I} \bar{g}_{k i a b} \leq f_{k a b}, \quad k \in K ; a \in A ; b \in B L \\
& \sum_{k \in K} \bar{g}_{k i a b} \\
& \geq s s_{i a b}+\sum_{h \in H}\left(w_{i h a b}+\sum_{b^{\prime} \in B L, b^{\prime} \neq b} q_{b^{\prime} b} \cdot w^{\prime}{ }_{i h a b^{\prime} b}\right), \\
& i s_{i a b} \geq \sum_{h \in H}\left(z_{i h a b}+\sum_{b^{\prime} \in B L, b^{\prime} \neq b} q_{b^{\prime} b} \cdot z^{\prime}{ }_{i h a b^{\prime} b}\right),
\end{aligned}
$$$$
i \in I ; a \in A ; b \in B L
$$

$$
\begin{aligned}
& \sum_{i \in I}\left(z_{i h a b}+\sum_{b^{\prime} \in B L, b^{\prime} \neq b} q_{b b^{\prime}} \cdot z_{i h a b b^{\prime}}^{\prime}\right) \\
& \quad \geq \sum_{i \in I}\left(x_{i} \cdot \Delta T \cdot \bar{d}_{h a b}\right), \quad h \in H ; a \in A ; b \in B L
\end{aligned}
$$

Let $\bar{g}_{k i a b}$ be the expected distribution flow for blood product $a$ with type $b$ from donor point $k$ to blood bank candidate $i$. Constraints (37) enforce that the total expected quantity of blood provided by donor points within each $T$, which involves emergency stock, is not more than the maximum inventory in corresponding blood bank. Constraints
TABLE 23: The one-to-one match between potential epicenters and hospitals.

\begin{tabular}{lc}
\hline Potential epicenters & Hospitals \\
\hline Wenchuan & WPH \\
Beichuan & $\mathrm{BPH}$ \\
Maoxian & $\mathrm{WPH}$ \\
Lushan & $\mathrm{WCH}$ \\
Pingwu & $\mathrm{BPH}$ \\
\hline
\end{tabular}

(38) express the capacity of blood supply by each donor point. Constraints (39) specify that the quantity of daily and emergency blood supplied to hospitals should be less than the quantity provided by donor points within each $T$. Constraints (40) ensure that enough blood is prepositioned in blood bank for an earthquake. Decision variables $z_{i h a b}$ and $z^{\prime}{ }_{i h a b}{ }^{\prime} b$ are the quantities of emergency blood supply for corresponding blood type $b$ and type $b^{\prime}$ which can be substituted by type $b$, respectively. Constraints (41) show that the additional quantity of blood supply should satisfy the expected value of demand caused by the earthquakes, in which $\bar{d}_{h a b}$ is the expected value of demand caused by the earthquake for blood product $a$ with type $b$ in hospital $h$. In summary, the $\mathrm{EV}$ model is a mixed-integer linear programming which is tractable. The key is to give the expected emergency demand which would be shown at the next section.

5.2. The Simulation Parameters of the EV Model. Taking the dataset 1200_1 in Section 4.1 as the example, we next show how the parameters, including the expected demand, in the EV model can be given.

At first, according to the distance from epicenters to hospitals in Table 12, Table 23 shows the one-to-one match between potential epicenters and hospitals. In the EV model, we assume that four hospitals are all available in the disaster.

According to the probabilities of four magnitudes, formulas (42) show the expected magnitude, and (43) give the mean intensity according to formulas (27), respectively.

$$
\begin{aligned}
\overline{F_{m}}= & 0.264 \times 8+0.189 \times 7+0.151 \times 6.5 \\
& +0.396 \times 6=6.7925, \quad F_{m} \in F \\
\overline{\text { Indensity }}= & \frac{\overline{F_{m}}-1.5}{0.58}=9.125, \quad F_{m} \in F
\end{aligned}
$$

Then the total number of the injured can be calculated by formulas (28) and (29) by using the following two variables.

The proportions of two-level demand and four blood types are both turned into corresponding expected value. 
TABLE 24: The average blood demand (U) of each epicenter.

\begin{tabular}{|c|c|c|c|c|c|c|}
\hline \multirow{2}{*}{ Blood product } & \multirow{2}{*}{ Blood type } & \multicolumn{5}{|c|}{ Epicenters } \\
\hline & & Wenchuan & Beichuan & Maoxian & Lushan & Pingwu \\
\hline \multirow{4}{*}{ Plasma } & A & 282.28 & 645.10 & 291.22 & 775.02 & 317.68 \\
\hline & B & 217.14 & 496.23 & 224.01 & 596.17 & 244.37 \\
\hline & $\mathrm{AB}$ & 65.14 & 148.87 & 67.20 & 178.85 & 73.31 \\
\hline & $\mathrm{O}$ & 304.00 & 694.72 & 313.62 & 834.63 & 342.12 \\
\hline \multirow{4}{*}{ Red blood cells } & A & 1180.22 & 2697.16 & 1217.58 & 3240.33 & 1328.23 \\
\hline & B & 907.86 & 2074.74 & 936.60 & 2492.56 & 1021.71 \\
\hline & $\mathrm{AB}$ & 272.36 & 622.42 & 280.98 & 747.77 & 306.51 \\
\hline & $\mathrm{O}$ & 1271.01 & 2904.64 & 1311.24 & 3489.59 & 1430.40 \\
\hline \multirow{4}{*}{ Platelets } & $\mathrm{A}$ & 199.88 & 456.79 & 206.21 & 548.78 & 224.95 \\
\hline & B & 153.75 & 351.37 & 158.62 & 422.14 & 173.04 \\
\hline & $\mathrm{AB}$ & 46.13 & 105.41 & 47.59 & 126.64 & 51.91 \\
\hline & $\mathrm{O}$ & 215.26 & 491.92 & 222.07 & 590.99 & 242.25 \\
\hline
\end{tabular}

According to the proportion of two-level demand, blood type distribution and the corresponding probability stated in Section 4.1, let 0.35:0.65 be the mean proportion of twolevel injury and $p_{\mathrm{A}}: p_{\mathrm{B}}: p_{\mathrm{AB}}: p_{\mathrm{O}}=0.325: 0.25: 0.075: 0.35$ be the distribution proportion of four types (A: $\mathrm{B}: \mathrm{AB}: \mathrm{O})$. Same with the assumption in the two-stage SP, the blood bank is only required to meet the emergency blood demand in 2 hours. According to the expected proportions above, Tables 16 and 24 show the expected blood demand for each epicenter.

Multiplying the expected blood demand (see Table 24) by the corresponding probability of single-point earthquake (see Table 14), Table 25 is obtained in which the daily blood demand and expected emergency blood demand for each hospital are shown.

The parameters of the EV models under other datasets can be obtained in the same way.

5.3. The EV Solution and Its Analysis. Based on above parameters, IBM ILOG CPLEX 12.6 .3 is used to optimally solve the corresponding EV model. In this EV solution, the central blood bank is located in Chengdu, and the total cost is $4.86 \times 10^{7}(\mathrm{RMB})$. The maximum inventory and emergency stock of Expected Value Model are shown in Table 26.

By comparing the results of the RP and EV solution, the EV solution performs better in terms of the total cost. However, the EV model, which is under the deterministic setting, only takes the expected demand into account. It is necessary to validate its performance under the disaster scenarios [40].

Thus, for each dataset as shown in Table 18, we bring the EV solution into each scenario to check whether blood shortage occurs. The performance of the EV solution can be evaluated by the total probability of blood shortage occurrence if disasters really happen. We name it as failure probability (FP).

At first, the FP results under different disaster probabilities are displayed in Table 27. It is found that all the EV solutions would lead to poor FP results. When we consider higher disaster probability, the more emergency stock would be prepositioned in the EV solution, and then the corresponding FP would be improved but also larger than $65 \%$.

Furthermore, as to the UIH cost, its change would not influence the EV model's decisions on inventory because they are actually decided by the expected disaster demand. Hence, the FP results would not vary with the UIH cost and their values are same with the case of the disaster probability change ratio 1 as shown in Table 27.

In summary, compared with the EV solution, the RP solution can ensure the supply while reducing cost as possible by taking all possible disaster scenarios into account. Hence, the SP model which can generate the RP solution has the significant value for the blood management in the setting of disasters.

\section{Conclusions}

Human blood is a crucial and precious relief good. In this paper, we proposed a BN-based two-stage SP model to study the BLN planning problem, taking the suddenly-occurring disasters into account. The planning decisions in the BLN and the long-term operational decisions under the setting of daily and disaster scenarios are optimized globally by the two-stage SP model. The stochastic scenarios used in the SP model is generated by the proposed $\mathrm{BN}$ model in which multiple disaster-related factors that contribute the emergency blood demand are considered in a systematic way. Based on earthquakes in Sichuan Province, China, the simulations and related sensitivity analysis are implemented. Several observations and corresponding implications can be obtained from the results.

First, the BNs are able to describe the disaster scenarios and thus, work with the SP model to generate the effective solutions. Results indicate all these solutions significantly outperform the corresponding EV solutions in terms of the effects of rescue. Moreover, the scenario set with different dimensionalities can give the consistent location results. Hence, it is important and necessary to put efforts on the modelling of the BNs. 
TABLE 25: The daily demands and expected emergency demands caused by the earthquake in hospital $h$.

\begin{tabular}{|c|c|c|c|c|c|c|}
\hline & \multirow{2}{*}{ Blood product } & \multirow{2}{*}{ Blood type } & \multicolumn{4}{|c|}{ Hospitals } \\
\hline & & & $\mathrm{WCH}$ & $\mathrm{BPH}$ & WPH & $\mathrm{MPH}$ \\
\hline \multirow{12}{*}{$\begin{array}{l}\text { Daily } \\
\text { demands } \\
d_{\text {hab }} \\
(\mathrm{U} / \mathrm{h})\end{array}$} & \multirow{4}{*}{ Plasma } & A & 3.050 & 0.213 & 0.081 & 1.191 \\
\hline & & B & 2.644 & 0.184 & 0.070 & 1.032 \\
\hline & & $\mathrm{AB}$ & 0.718 & 0.050 & 0.019 & 0.280 \\
\hline & & $\mathrm{O}$ & 3.007 & 0.281 & 0.107 & 1.562 \\
\hline & \multirow{4}{*}{$\begin{array}{l}\text { Red blood } \\
\text { cells }\end{array}$} & $\mathrm{A}$ & 1.932 & 0.137 & 0.052 & 0.755 \\
\hline & & B & 1.675 & 0.118 & 0.045 & 0.655 \\
\hline & & $\mathrm{AB}$ & 0.455 & 0.032 & 0.012 & 0.178 \\
\hline & & $\mathrm{O}$ & 2.532 & 0.179 & 0.068 & 0.989 \\
\hline & \multirow{4}{*}{ Platelets } & A & 0.829 & 0.058 & 0.022 & 0.324 \\
\hline & & $\mathrm{B}$ & 0.719 & 0.050 & 0.019 & 0.281 \\
\hline & & $\mathrm{AB}$ & 0.195 & 0.013 & 0.005 & 0.076 \\
\hline & & $\mathrm{O}$ & 1.087 & 0.076 & 0.029 & 0.425 \\
\hline \multirow{12}{*}{$\begin{array}{l}\text { Expected } \\
\text { emergency } \\
\text { demands } \\
\Delta T \cdot \bar{d}_{\text {hab }} \\
\text { (U) }\end{array}$} & \multirow{4}{*}{ Plasma } & $\mathrm{A}$ & 10.850 & 17.696 & 13.437 & 0 \\
\hline & & $\mathrm{B}$ & 8.346 & 13.613 & 10.336 & 0 \\
\hline & & $\mathrm{AB}$ & 2.504 & 4.084 & 3.101 & 0 \\
\hline & & $\mathrm{O}$ & 11.685 & 19.058 & 14.471 & 0 \\
\hline & \multirow{4}{*}{$\begin{array}{l}\text { Red blood } \\
\text { cells }\end{array}$} & A & 45.365 & 73.989 & 56.180 & 0 \\
\hline & & $\mathrm{B}$ & 34.896 & 56.914 & 43.216 & 0 \\
\hline & & $\mathrm{AB}$ & 10.469 & 17.074 & 12.965 & 0 \\
\hline & & $\mathrm{O}$ & 48.854 & 79.680 & 60.502 & 0 \\
\hline & \multirow{4}{*}{ Platelets } & A & 7.683 & 12.531 & 9.515 & 0 \\
\hline & & $\mathrm{B}$ & 5.910 & 9.639 & 7.319 & 0 \\
\hline & & $\mathrm{AB}$ & 1.773 & 2.892 & 2.196 & 0 \\
\hline & & $\mathrm{O}$ & 8.274 & 13.494 & 10.246 & 0 \\
\hline
\end{tabular}

TABLE 26: The decisions of maximum inventory and emergency stock of the blood bank in the EV solution.

\begin{tabular}{|c|c|c|c|c|c|c|c|}
\hline \multicolumn{8}{|c|}{ The prespecified central blood bank: Chengdu } \\
\hline \multirow{12}{*}{$\begin{array}{c}\text { Maximum } \\
\text { inventory } \\
C_{i a b}(\mathrm{U})\end{array}$} & \multirow{4}{*}{ Plasma } & $\mathrm{A}$ & 807.29 & \multirow{12}{*}{$\begin{array}{c}\text { Emergency } \\
\text { stock } \\
s s_{i a b}(\mathrm{U})\end{array}$} & \multirow{4}{*}{ Plasma } & $\mathrm{A}$ & 42 \\
\hline & & $\mathrm{B}$ & 695.67 & & & B & 33 \\
\hline & & $\mathrm{AB}$ & 189.74 & & & $\mathrm{AB}$ & 10 \\
\hline & & $\mathrm{O}$ & 882.33 & & & $\mathrm{O}$ & 46 \\
\hline & \multirow{4}{*}{ Red blood cells } & A & 660.87 & & \multirow{4}{*}{ Red blood cells } & A & 176 \\
\hline & & $\mathrm{B}$ & 555.74 & & & $\mathrm{~B}$ & 136 \\
\hline & & $\mathrm{AB}$ & 154.74 & & & $\mathrm{AB}$ & 41 \\
\hline & & $\mathrm{O}$ & 824.9 & & & $\mathrm{O}$ & 190 \\
\hline & \multirow{4}{*}{ Platelets } & $\mathrm{A}$ & 237.79 & & \multirow{4}{*}{ Platelets } & A & 30 \\
\hline & & $\mathrm{B}$ & 203.26 & & & $\mathrm{~B}$ & 23 \\
\hline & & $\mathrm{AB}$ & 55.63 & & & $\mathrm{AB}$ & 7 \\
\hline & & $\mathrm{O}$ & 304.89 & & & $\mathrm{O}$ & 33 \\
\hline
\end{tabular}

Furthermore, the larger scenario set can cover more detailed disaster scenarios, but it does not mean the bigger dimensionality is better. Because it not only requires more modelling efforts but also may cause conservative solutions, such as more prepositioning inventory. Considering the preciousness and perishability of human blood, over-stock also should be avoided.
At last, the parameters, including the random factors involving the BNs and the deterministic ones, would influence the final decisions in different ways. For example, the total quantity of the emergency stock is only influenced by the some of the BN-related factors, while the decision on blood bank location is decided by the cost-related parameters, such as the unit inventory holding cost. Hence, by the utilization 
TABLE 27: The FP of the EV solutions of each dataset under varying disaster probabilities.

\begin{tabular}{|c|c|c|c|c|c|c|c|c|c|}
\hline \multirow{2}{*}{$\begin{array}{l}\text { The disaster probability } \\
\text { change ratio }\end{array}$} & \multicolumn{6}{|c|}{1200} & \multicolumn{2}{|c|}{3600} & \multirow{2}{*}{ 14500_ } \\
\hline & 1200_1 & 1200_2 & 1200_3 & 1200_4 & 1200_5 & 1200_6 & 3600_1 & $3600 \_2$ & \\
\hline 0.2 & $83.92 \%$ & $84.30 \%$ & $84.30 \%$ & $83.92 \%$ & $83.92 \%$ & $83.92 \%$ & $83.80 \%$ & $83.80 \%$ & $83.95 \%$ \\
\hline 0.25 & $84.08 \%$ & $84.08 \%$ & $84.08 \%$ & $84.08 \%$ & $84.08 \%$ & $84.08 \%$ & $84.06 \%$ & $84.06 \%$ & $84.22 \%$ \\
\hline 0.5 & $83.93 \%$ & $83.93 \%$ & $83.93 \%$ & $83.93 \%$ & $83.93 \%$ & $83.93 \%$ & $83.95 \%$ & $83.95 \%$ & $83.97 \%$ \\
\hline 1 & $83.98 \%$ & $83.98 \%$ & $83.98 \%$ & $64.80 \%$ & $64.80 \%$ & $77.43 \%$ & $78.61 \%$ & $77.39 \%$ & $77.45 \%$ \\
\hline 2 & $64.80 \%$ & $64.80 \%$ & $64.80 \%$ & $64.80 \%$ & $64.80 \%$ & $64.80 \%$ & $64.83 \%$ & $64.83 \%$ & $64.80 \%$ \\
\hline 3 & $64.96 \%$ & $64.96 \%$ & $64.96 \%$ & $64.96 \%$ & $64.96 \%$ & $64.96 \%$ & $64.96 \%$ & $64.96 \%$ & $64.97 \%$ \\
\hline 4 & $65.30 \%$ & $65.30 \%$ & $65.30 \%$ & $65.30 \%$ & $65.30 \%$ & $65.30 \%$ & $65.31 \%$ & $65.31 \%$ & $65.31 \%$ \\
\hline 5 & $65.30 \%$ & $65.30 \%$ & $65.30 \%$ & $65.30 \%$ & $65.30 \%$ & $65.30 \%$ & $65.29 \%$ & $65.29 \%$ & $65.30 \%$ \\
\hline
\end{tabular}

of the BNs, we can reveal the specific relationship between the random factors and the final decisions. For example, if the decision-maker cares about the planning of the total inventory, we should focus on the estimation of the severity of the injured, instead of the distribution of blood types, the disaster probability, and the unit inventory cost, etc. Thus, we can identify the specific efforts that should be made in terms of different planning purposes. This is the main benefit of the BNs.

There are some future directions as follows. At first, the $\mathrm{BN}$ model we proposed here only fits earthquakes. The applications of the BNs in the setting of other disasters still leave lots of opportunities. Moreover, we only consider the uncertainty of demand in this paper. But, when the earthquake occurs, blood shortage may be satisfied by urgent blood donations partially which are also random. And the more serious the disaster is, the more relief goods, including blood, the government and the people donate. Alfonso et al. [55], Jabbarzadeh et al. [22], and Chaiwuttisak et al. [56] notice the uncertainty of the donations but fail to utilize its underlying relationship with the random demand. The $\mathrm{BN}$ is still a possible way to model such relationships which can be addressed in future research. At last, another interesting research direction is to explore the application of distributionally robust approach which only requires moments information [57-59]. But the underlying correlations between different stochastic variables, which are involved in the second moment, usually are hard to obtained [32]. A natural idea is to address this issue by the $\mathrm{BN}$.

\section{Data Availability}

The data used to support the findings of this study are available from the corresponding author upon request.

\section{Conflicts of Interest}

The authors declare that there are no conflicts of interest regarding the publication of this paper.

\section{Acknowledgments}

This work is supported by National Natural Science Foundation of P. R. China (Grant Nos. 71572033 and 71832001).

\section{References}

[1] M. Rezaei-Malek, R. Tavakkoli-Moghaddam, B. Zahiri, and A. Bozorgi-Amiri, "An interactive approach for designing a robust disaster relief logistics network with perishable commodities," Computers \& Industrial Engineering, vol. 94, no. C, pp. 201-215, 2016.

[2] M. Eskandari-Khanghahi, R. Tavakkoli-Moghaddam, A. A. Taleizadeh, and S. H. Amin, "Designing and optimizing a sustainable supply chain network for a blood platelet bank under uncertainty," Engineering Applications of Artificial Intelligence, vol. 71, pp. 236-250, 2018.

[3] H. Mo, World Disasters Report, 2015, http://ifrc-media.org/ interactive/world-disasters-report-2015/.

[4] J. B. Sheu, "Dynamic relief-demand management for emergency logistics operations under large-scale disasters," Transportation Research Part E: Logistics and Transportation Review, vol. 46, no. 1, pp. 1-17, 2010.

[5] B. Vahdani, M. Soltani, M. Yazdani, and S. Meysam Mousavi, "A three level joint location-inventory problem with correlated demand, shortages and periodic review system: robust metaheuristics," Computers \& Industrial Engineering, vol. 109, no. 7, pp. 113-129, 2017.

[6] J. Beliën and H. Forcé, "Supply chain management of blood products: a literature review," European Journal of Operational Research, vol. 217, no. 1, pp. 1-16, 2012.

[7] Q. Duan and T. W. Liao, "Optimization of blood supply chain with shortened shelf lives and $\mathrm{ABO}$ compatibility," International Journal of Production Economics, vol. 153, no. 7, pp. 113-129, 2014.

[8] D. Alem, A. Clark, and A. Moreno, "Stochastic network models for logistics planning in disaster relief," European Journal of Operational Research, vol. 255, no. 1, pp. 187-206, 2016.

[9] G. Galindo and R. Batta, "Review of recent developments in OR/MS research in disaster operations management," European Journal of Operational Research, vol. 230, no. 2, pp. 201-211, 2013.

[10] J. A. Paul and L. MacDonald, "Location and capacity allocations decisions to mitigate the impacts of unexpected disasters," European Journal of Operational Research, vol. 251, no. 1, pp. 252-263, 2016. 
[11] G. Barbarosoğlu and Y. Arda, "A two-stage stochastic programming framework for transportation planning in disaster response," Journal of the Operational Research Society, vol. 55, no. 1, pp. 43-53, 2004.

[12] H. O. Mete and Z. B. Zabinsky, "Stochastic optimization of medical supply location and distribution in disaster management," International Journal of Production Economics, vol. 126, no. 1, pp. 76-84, 2010.

[13] M. Haghi, S. M. T. Fatemi Ghomi, and F. Jolai, "Developing a robust multi-objective model for pre/post disaster times under uncertainty in demand and resource," Journal of Cleaner Production, vol. 154, pp. 188-202, 2017.

[14] S. Tofighi, S. A. Torabi, and S. A. Mansouri, "Humanitarian logistics network design under mixed uncertainty," European Journal of Operational Research, vol. 250, no. 1, pp. 239-250, 2016.

[15] A. Mohamadi and S. Yaghoubi, "A bi-objective stochastic model for emergency medical services network design with backup services for disasters under disruptions: an earthquake case study," International Journal of Disaster Risk Reduction, vol. 23, pp. 204-217, 2017.

[16] S. An, N. Cui, Y. Bai, W. Xie, M. Chen, and Y. Ouyang, "Reliable emergency service facility location under facility disruption, en-route congestion and in-facility queuing," Transportation Research Part E: Logistics and Transportation Review, vol. 82, pp. 199-216, 2015.

[17] A. Verma and G. M. Gaukler, "Pre-positioning disaster response facilities at safe locations: an evaluation of deterministic and stochastic modeling approaches," Computers \& Operations Research, vol. 62, pp. 197-209, 2015.

[18] C. G. Kochan, S. S. Kulkarni, and D. R. Nowicki, "Efficient inventorying and distribution of blood product during disasters," in Advances in Managing Humanitarian Operations, International Series in Operations Research \& Management Science, pp. 185-204, Springer International Publishing, Cham, Switzerland, 2016.

[19] S. Gunpinar and G. Centeno, "Stochastic integer programming models for reducing wastages and shortages of blood products at hospitals," Computers \& Operations Research, vol. 54, no. 54, pp. 129-141, 2015.

[20] B. Fahimnia, A. Jabbarzadeh, A. Ghavamifar, and M. Bell, "Supply chain design for efficient and effective blood supply in disasters," International Journal of Production Economics, vol. 183, pp. 700-709, 2017.

[21] M. R. Ghatreh Samani, S. A. Torabi, and S.-M. HosseiniMotlagh, "Integrated blood supply chain planning for disaster relief," International Journal of Disaster Risk Reduction, vol. 27, pp. 168-188, 2018.

[22] A. Jabbarzadeh, B. Fahimnia, and S. Seuring, "Dynamic supply chain network design for the supply of blood in disasters: a robust model with real world application," Transportation Research Part E: Logistics and Transportation Review, vol. 70, no. 1, pp. 225-244, 2014.

[23] S. Khalilpourazari and A. Arshadi Khamseh, "Bi-objective emergency blood supply chain network design in earthquake considering earthquake magnitude: a comprehensive study with real world application," Annals of Operations Research, no. 1, pp. 1-39, 2017.

[24] F. Salehi, M. Mahootchi, and S. M. Husseini, "Developing a robust stochastic model for designing a blood supply chain network in a crisis: a possible earthquake in Tehran," Annals of Operations Research, pp. 1-25, 2017.
[25] D. Rahmani, "Designing a robust and dynamic network for the emergency blood supply chain with the risk of disruptions," Annals of Operations Research, no. 1, pp. 1-29, 2018.

[26] D. Shishebori and A. Yousefi Babadi, "Robust and reliable medical services network design under uncertain environment and system disruptions," Transportation Research Part E: Logistics and Transportation Review, vol. 77, pp. 268-288, 2015.

[27] B. Zahiri, S. A. Torabi, M. Mousazadeh, and S. A. Mansouri, "Blood collection management: methodology and application," Applied Mathematical Modelling: Simulation and Computation for Engineering and Environmental Systems, vol. 39, no. 23-24, pp. 7680-7696, 2015.

[28] M. Fereiduni and K. Shahanaghi, "A robust optimization model for blood supply chain in emergency situations," International Journal of Industrial Engineering Computations, vol. 7, no. 4, pp. 535-554, 2016.

[29] R. Ramezanian and Z. Behboodi, "Blood supply chain network design under uncertainties in supply and demand considering social aspects," Transportation Research Part E: Logistics and Transportation Review, vol. 104, pp. 69-82, 2017.

[30] M. R. G. Samani and S.-M. Hosseini-Motlagh, "An enhanced procedure for managing blood supply chain under disruptions and uncertainties," Annals of Operations Research, no. 1, pp. 150, 2018.

[31] A. Kamyabniya, M. M. Lotf, M. Naderpour, and Y. Yih, "Robust platelet logistics planning in disaster relief operations under uncertainty: a coordinated approach," Information Systems Frontiers, vol. 20, no. 4, pp. 759-782, 2018.

[32] S. Agrawal, Y. Ding, A. Saberi, and Y. Ye, "Price of correlations in stochastic optimization," Operations Research, vol. 60, no. 1, pp. 150-162, 2012.

[33] V. Gabrel, C. Murat, and A. Thiele, "Recent advances in robust optimization: an overview," European Journal of Operational Research, vol. 235, no. 3, pp. 471-483, 2014.

[34] N. Bassamzadeh and R. Ghanem, "Multiscale stochastic prediction of electricity demand in smart grids using Bayesian networks," Applied Energy, vol. 193, pp. 369-380, 2017.

[35] F. Archetti, A. Gaivoronski, and F. Stella, "Stochastic optimization on Bayesian nets," European Journal of Operational Research, vol. 101, no. 2, pp. 360-373, 1997.

[36] J. Wu, S. Xu, R. Zhou, and Y. Qin, "Scenario analysis of mine water inrush hazard using Bayesian networks," Safety Science, vol. 89, pp. 231-239, 2016.

[37] M. Afenyo, F. Khan, B. Veitch, and M. Yang, "Arctic shipping accident scenario analysis using Bayesian Network approach," Ocean Engineering, vol. 133, pp. 224-230, 2017.

[38] Y. Huang, G. Ma, and J. Li, "Grid-based risk mapping for gas explosion accidents by using Bayesian network method," Journal of Loss Prevention in the Process Industries, vol. 48, pp. 223-232, 2017.

[39] A. Sebastian, E. J. C. Dupuits, and O. Morales-Nápoles, “Applying a Bayesian network based on Gaussian copulas to model the hydraulic boundary conditions for hurricane flood risk analysis in a coastal watershed," Coastal Engineering Journal, vol. 125, pp. 42-50, 2017.

[40] J. R. Birge and F. V. Louveaux, Introduction to Stochastic Programming, Springer, London, UK, 2011.

[41] Y. X. Hu, Earthquake Engineering, Seismological Press, Beijing, China, 1988.

[42] U. Chandra, "Attenuation of intensities in the united states," Bulletin of the Seismological Society of America, vol. 69, no. 6, pp. 2003-2024, 1979. 
[43] S. P. Lee, "Practical scale," Chinese Journal of Geophysics, vol. 7, no. 2, pp. 98-102, 1958.

[44] X. Ma, Study on the Location and Allocation of Social Emergency Materials Reservation, Graduate University of Chinese Academy of Sciences, Beijing, China, 2011.

[45] M. Wyss and G. Trendafiloski, "Trends in the casualty ratio of injured to fatalities in earthquakes," in Advances in Natural and Technological Hazards Research, R. Spence, E. So, and C. Scawthorn, Eds., vol. 29, Springer, Dordrecht, Netherlands, 2011.

[46] D. R. Peng, "The distribution of ABO blood group of Han nationality in China," Chinese Journal of Blood Transfusion, vol. 4, no. 1, pp. 20-23, 1991.

[47] Z. Y. Chen, "The distribution of ABO blood group in Chinese," Hereditas, vol. 4, no. 2, pp. 4-7, 1982.

[48] World Population Network, "Ranking of Sichuan Population," 2014, http://www.renkou.org.cn/china/sichuan/2014/2208.html.

[49] The Minister of Health of the People's Republic of China, People's Republic of China Health Industry Standard (Standards for Blood Storage), People's Republic of China, 2012, http://www.csbt.org.cn/plus/view.php?aid=94.

[50] X. L. Deng, X. H. Liang, L. Wang et al., "Survey on the situation of blood supply in 355 blood supply institutions," Chinese Journal of Blood Transfusion, vol. 24, no. 9, pp. 779-780, 2011.

[51] J. Wang, L. Jiang, and Y. H. Wang, "Analysis of clinical blood and its significance in general hospital," Psychologist, vol. 22, no. 30, pp. 31-32, 2016.

[52] Z.-T. Liu, D.-L. Wang, W.-J. Zhang, W. Feng, and T.-Y. Zheng, "Early judgment method of earthquake emergency response level based on Bayesian classification," Earthquake, vol. 31, no. 2, pp. 114-121, 2011.

[53] J. Ling, Y. He, X. Q. Liu et al., "Analysis of characteristics about blood transfusion in 2065 cases of inpatients injured by earthquake," Laboratory Medicine and Clinic, vol. 7, no. 1, pp. 78, 2010.

[54] C. H. Liu, C. L. Yuan, and Q. J. Huang, "Analysis of features of 320 blood transfusion earthquake victims in Deyang area," Laboratory Medicine and Clinic, vol. 7, no. 1, pp. 11-12, 2010.

[55] E. Alfonso, X. Xie, and V. Augusto, "A simulation-optimization approach for capacity planning and appointment scheduling of blood donors based on mathematical programming representation of event dynamics," in Proceedings of the 11th IEEE International Conference on Automation Science and Engineering, CASE 2015, pp. 728-733, Sweden, August 2015.

[56] P. Chaiwuttisak, H. Smith, Y. Wu, C. Potts, T. Sakuldamrongpanich, and S. Pathomsiri, "Location of low-cost blood collection and distribution centres in Thailand," Operations Research for Health Care, vol. 9, pp. 7-15, 2016.

[57] E. Delage and Y. Ye, "Distributionally robust optimization under moment uncertainty with application to data-driven problems," Operations Research, vol. 58, no. 3, pp. 595-612, 2010.

[58] D. Bertsimas, K. Natarajan, and C.-P. Teo, "Probabilistic combinatorial optimization: moments, semidefinite programming and asymptotic bounds," SIAM Journal on Optimization, vol. 15, no. 1, pp. 185-209, 2004.

[59] D. Bertsimas, X. V. Doan, K. Natarajan, and C.-P. Teo, "Models for minimax stochastic linear optimization problems with risk aversion," Mathematics of Operations Research, vol. 35, no. 3, pp. 580-602, 2010. 


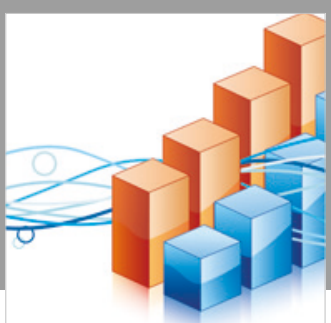

Advances in

Operations Research

\section{-n-m}
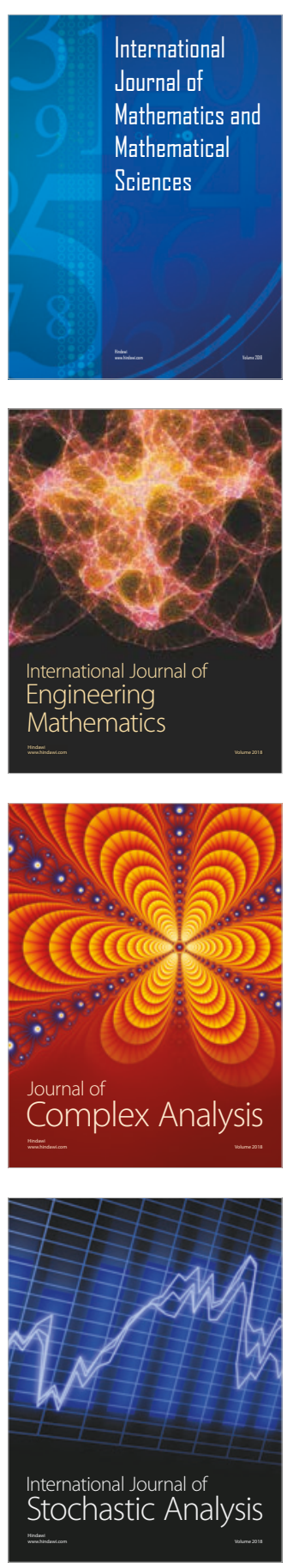
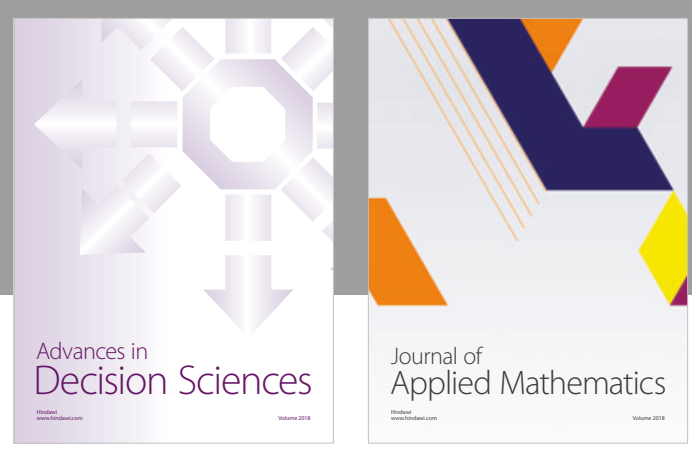

Journal of

Applied Mathematics
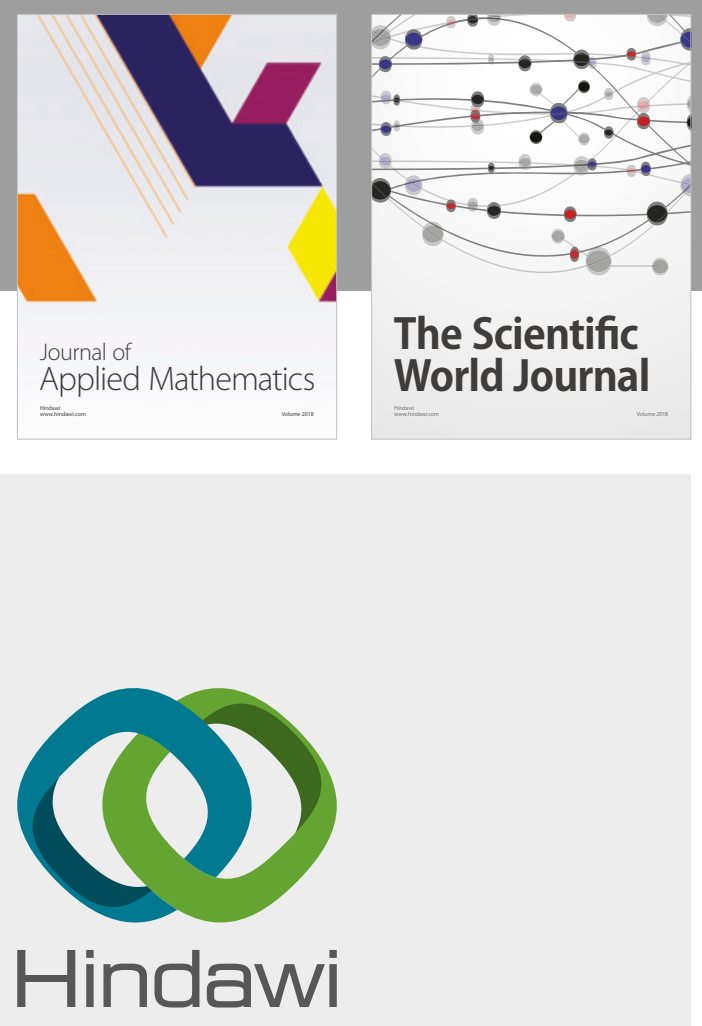

Submit your manuscripts at

www.hindawi.com

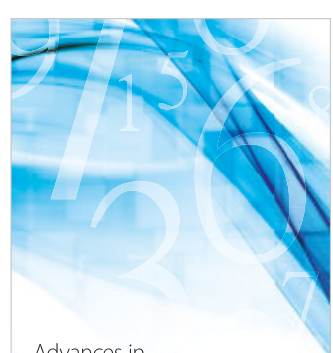

Advances in
Numerical Analysis
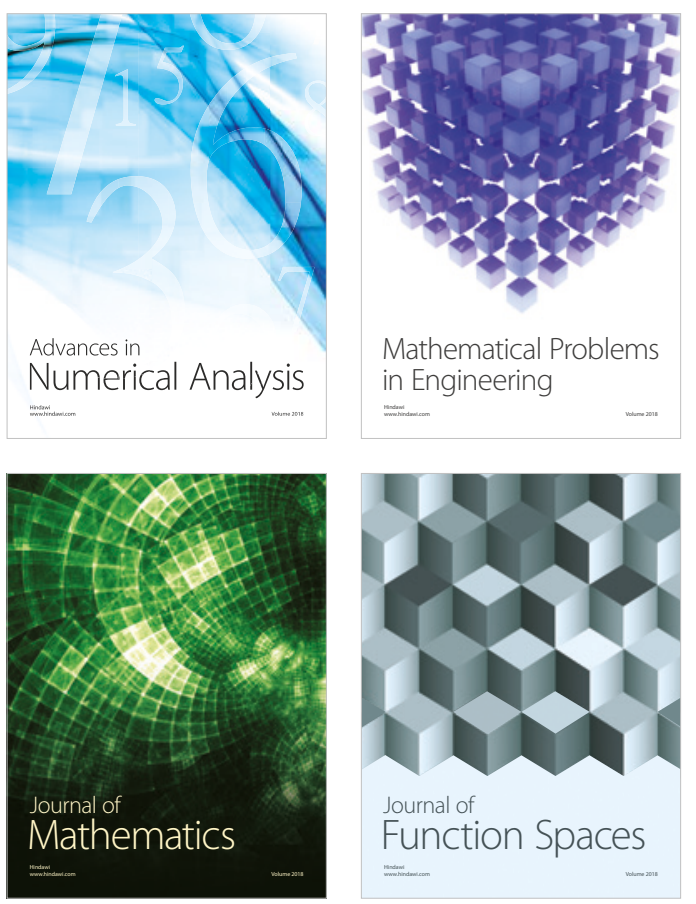

Mathematical Problems in Engineering

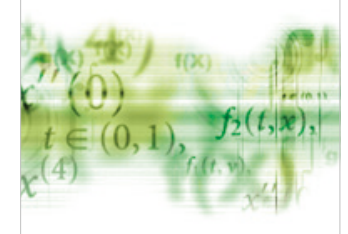

International Journal of

Differential Equations

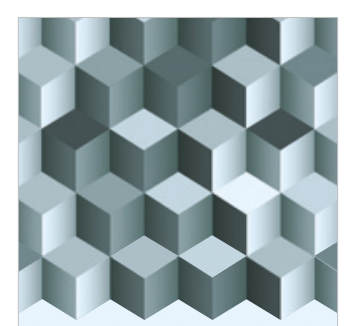

Journal of

Function Spaces

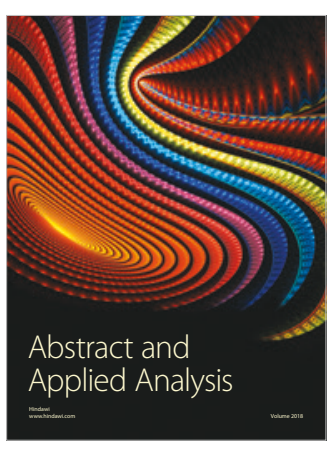

The Scientific

World Journal

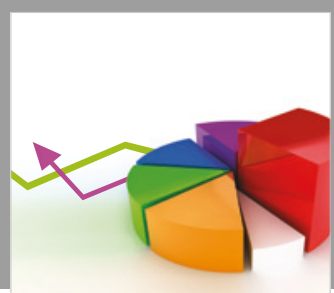

Journal of

Probability and Statistics
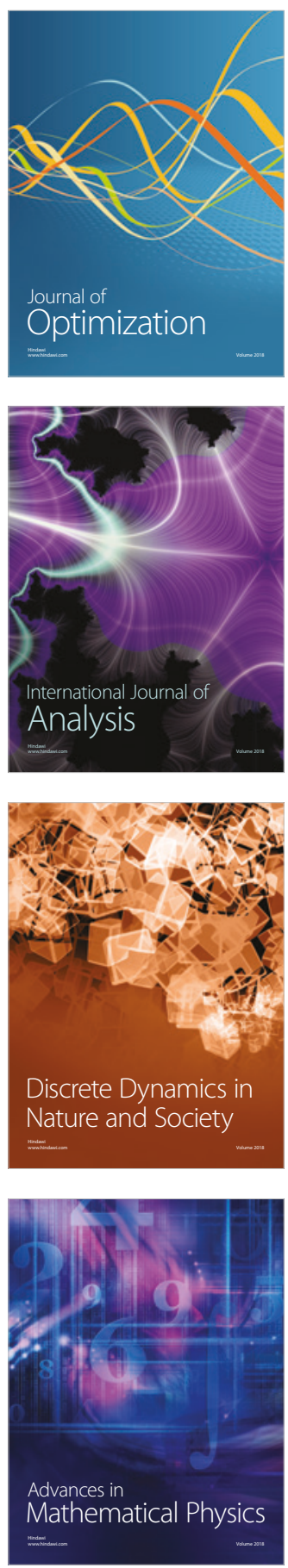